\title{
Australian Asian Options
}

\author{
Manuel Moreno \\ Department of Economics and Business \\ Universitat Pompeu Fabra \\ Carrer Ramon Trias Fargas, 25-27 \\ 08005 Barcelona, Spain \\ phone: +34 935422771 \\ fax: $\quad+34935421746$ \\ e-mail: manuel.moreno@econ.upf.es
}

\author{
Javier F. Navas \\ Department of Finance \\ Instituto de Empresa \\ María de Molina, 13 \\ 28006 Madrid, Spain \\ phone: +34 915689600 \\ fax: $\quad+34915636859$ \\ e-mail: jfnavas@ie.edu
}

This Version: February 25, 2003

\section{Acknowledgments}

We would like to thank Arturo Kohatsu and seminar participants at the Universidad Pablo de Olavide, Universitat Pompeu Fabra and Universidad de Navarra for their valuable suggestions and comments. The first author gratefully acknowledges financial support by DGESIC grant BEC2002-00429. The second author thanks the hospitality of the Universitat Pompeu Fabra, where part of this work was done. The usual caveat applies. 


\title{
Australian Asian Options
}

\begin{abstract}
We study European options on the ratio of the stock price to its average and viceversa. Some of these options are traded in the Australian Stock Exchange since 1992, thus we call them Australian Asian options. For geometric averages, we obtain closed-form expressions for option prices. For arithmetic means, we use different approximations that produce very similar results.
\end{abstract}

Keywords: Asian Options, Arithmetic Average, Geometric Average, Edgeworth Expansion, Lognormal Distribution, Gamma Distribution.

Journal of Economic Literature classification: G13, C15 


\section{Introduction}

Asian options are options on average values of asset prices. Their prices depend on the asset price history over the averaging period. Therefore, they are considered path-dependent claims.

These derivatives are traded in over-the-counter markets ${ }^{1}$ and it is usually argued that they provide the following advantages: (a) they are cheaper than standard European ones as the average is less volatile than the asset price itself, (b) this type of options prevents manipulation of the underlying asset price at the maturity date and (c) they are the adequate hedging instrument for traders who act continuously over finite periods.

Options on the ratio of the stock price to its average (or viceversa) are particular cases of Asian options. They have recently appeared as special types of variable purchase options (VPOs). VPOs were first issued in 1992 and have been traded since then on the Australian Stock Exchange. A VPO is an option that gives its holder the right to buy at maturity a stochastic number of shares that depends on the terminal stock price. This option can have more complex features like caps and floors on the number of shares. Handley (2000) provides a detailed description of VPOs as well as pricing formulae. He also describes Asian VPOs, in which the number of shares that can be bought at maturity depends on the average stock price. These options are shown to be equivalent to options on the ratio of the stock price to its average. Alternatively, we could define Asian VPOs in such a way that they are equivalent to options on the ratio of the average of the stock price to the stock price itself.

In this paper we price such options using both geometric and arithmetic 
(discrete- and continuous-time) means of stock prices that are assumed to follow a lognormal process. $^{2}$ When the average is computed on geometric basis, these ratios are lognormally distributed at maturity, thus we obtain Black-Scholes-type formulae to price options.

However, when the average is computed on arithmetic basis, the riskneutral distribution of these ratios is, in general, unknown and we can not obtain closed-form expressions for the prices of these options. ${ }^{3}$ This happens because the arithmetic average is the convolution of correlated lognormal random variables and its distribution is unknown. ${ }^{4}$

Some of the approaches that have been followed in the literature to study the pricing and hedging of arithmetic Asian options are:

- Numerical approximations

- Finite different schemes. In this case, a partial difference pricing equation is obtained and then solved numerically. See, for instance, Kemna and Vorst (1990), Rogers and Shi (1995), Alziari et al (1997) or Hansen and Jorgensen (2000). ${ }^{5}$

- Monte Carlo simulations. Here, variance reductions technique are commonly used to reduce standard errors. For example, Kemna and Vorst (1990) use the closed-form expressions for prices of geometric Asian options as control variables to price arithmetic ones with Monte Carlo. ${ }^{6}$

- General numerical methods. Some examples are the fast Fourier transform proposed in Carverhill and Clewlow (1990), the conditioning approach suggested in Curran (1994), Rogers and Shi (1995) and Nielsen 
and Sandmann (1996, 1999, 2001), and the accelerated simulation method presented in Vázquez-Abad and Dufresne (1998).

- Pseudo-analytic characterizations. this is the case of Yor $(1992,1993)$, Geman and Yor (1993), De Schepper et al (1994), Eydeland and Geman (1995), Fu et al (1999) and Shirakawa (1999), who use the theory of Bessel processes and the inversion of a Laplace transform. Alternatively, Ju (1997) employs A Fourier transform, while Dufresne (2000) suggests a Laguerre expansion.

- Analytical approximations. Jarrow and Rudd (1982) apply Edgeworth series expansion to the problem of option pricing when the risk-neutral distribution of the underlying asset at maturity is not known. Typically, up to fourth order moments are used to approximate the true probability density function. This method has been also used by Turnbull and Wakeman (1991), Ritchken et al (1993) and Jacques (1996). Some authors use only the first and second order moments in the Edgeworth series expansion, obtaining what is called the Wilkinson approximation. ${ }^{7}$ This is equivalent to assume that the true distribution is actually lognormal, so that Black-Scholes-type formulae can be used to price options. See Levy and Turnbull (1992) for a numerical comparison of the accuracy of these expansions. Other analytical approximations can be seen in Bouaziz et al (1994), Vorst (1990, 1992, 1996), Nielsen and Sandmann (1998), Posner and Milevsky (1998) and Chung et al (2001).

A different type of analytical approximation is presented in Milevsky 
and Posner (1998) who use the fact that the infinite sum of correlated lognormal random variables is reciprocal gamma distributed to obtain a closed-form solution for the value of arithmetic Asian options. ${ }^{8}$ This formula is exact only when the average is computed continuously.

In this paper we price arithmetic Australian Asian options using both the Wilkinson approximation and the gamma distribution. We also use Monte Carlo simulation with antithetic variables. The results show that option prices obtained with the three methods are quite similar. This is true even when the number of monitoring dates used to compute the average is small. Hence, in practice, it does not seem to be necessary to use higher order moments in the Edgeworth expansion nor to require a large number of monitoring dates in the gamma approximation to the option pricing problem.

The rest of the paper is organized as follows. Section 2 describes some statistical results that are used in the paper. In Section 3 we generalize the Black-Scholes formula for option prices. Section 4 presents closed-form expressions for the prices of geometric Australian Asian options and Section 5 presents approximations to the value of arithmetic ones. Finally, Section 6 summarizes and concludes.

\section{Previous Statistical Results}

The following Lemma specifies several features of the lognormal distribution that will be useful to find later results: 


\section{Lemma 1}

1. Let $Y=\ln (X)$ be a normal random variable with mean $m$ and variance $s^{2}$. Then, $X$ follows a lognormal distribution, that is, $X \sim \Lambda\left(m, s^{2}\right)$. Its density function is given by

$$
f(x)=\frac{1}{s x \sqrt{2 \pi}} \exp \left\{-\frac{1}{2}\left(\frac{\ln x-m}{s}\right)^{2}\right\}, x>0
$$

Moreover, it is verified that

$$
\begin{aligned}
E(X) & =\exp \left\{m+\frac{1}{2} s^{2}\right\} \\
V(X) & =[E(X)]^{2}\left[e^{s^{2}}-1\right] \\
E\left(X^{-1}\right) & =\exp \{-2 m\} E(X) \\
V\left(X^{-1}\right) & =\left[E\left(X^{-1}\right)\right]^{2}\left[e^{s^{2}}-1\right]
\end{aligned}
$$

2. The expectation of the truncated lognormal variable

$$
\tilde{X}=\left\{\begin{array}{c}
X \quad \text { if } \quad X \geq K \\
0 \quad \text { if } \quad X<K
\end{array}, \quad K \in \mathbb{R}^{+}\right.
$$

is given by

$$
E(\tilde{X})=E(X) N(s-D), \quad D=\frac{\ln K-m}{s}
$$

where $N($.$) denotes the distribution function of a standard normal vari-$ able.

Proof: See Appendix.

The following Lemma will be useful to compute the moments of ratios involving arithmetic average asset prices: 
Lemma 2 (M ood et al (1974))

Let $X$ and $Y$ be two random variables. Then, it is verified that

$$
\begin{aligned}
& E\left(\frac{X}{Y}\right) \simeq \frac{E(X)}{E(Y)}-\frac{1}{(E(Y))^{2}} \operatorname{Cov}(X, Y)+\frac{E(X)}{(E(Y))^{3}} V(Y) \\
& V\left(\frac{X}{Y}\right) \simeq\left(\frac{E(X)}{E(Y)}\right)^{2}\left(\frac{V(X)}{(E(X))^{2}}+\frac{V(Y)}{(E(Y))^{2}}-2 \frac{\operatorname{Cov}(X, Y)}{E(X) E(Y)}\right)
\end{aligned}
$$

Proof: See Mood et al, p. 181.

\section{The Generalized Black-Scholes Model}

Uncertainty is modelled by a filtered probability space $(\Omega, \mathbb{F}, P)$. The set of trading dates is $t \in[0, T]$. Let $Z=\left\{Z_{t} \mid t \in[0, T]\right\}$ be the price process for the underlying asset. The assumptions of the pricing model are as follows:

1. Markets are frictionless

(a) No transaction costs when trading the stock or the option

(b) No taxes

(c) No penalties to short selling

(d) All assets are perfectly divisible

2. Security trading is in continuous time

3. The term structure of interest rates is flat and known with certainty. Let $\beta=\left\{\beta_{t} ; t \in[0, T]\right\}$ be the value process for a banking account defined by

$$
d \beta_{t}=r \beta_{t} d t
$$

where $t \in[0, T], \beta_{0}=1$ and the risk-free interest rate, $r$, is constant 
4. The asset offers a continuous dividend yield of $\delta$ in the interval $[0, T]$

5. The asset price follows a GBM process

$$
d Z_{t}=\mu_{Z} Z_{t} d t+\sigma_{Z} Z_{t} d W_{t}
$$

where $\left(Z_{t}, t\right) \in(0, \infty) \times[0, T], \mu_{Z}$ and $\sigma_{Z}$ are constants and $W_{t}$ is a standard Wiener process. Usually, $\sigma_{Z}^{2}$ is referred to as the logarithmic variance parameter of the asset.

Under the risk-neutral probability measure, the process (7) becomes

$$
d Z_{t}=\alpha_{Z} Z_{t} d t+\sigma_{Z} Z_{t} d W_{t}
$$

where $\alpha_{Z}$ is the (constant) risk-neutral drift of the process.

The solution for this process is given by

$$
Z_{t}=Z_{0} \exp \left\{\left(\alpha_{Z}-\frac{1}{2} \sigma_{Z}^{2}\right) t+\sigma_{Z} W_{t}\right\}
$$

Therefore, $Z_{t}$ follows a lognormal process. Moreover, it is straightforward to show that

$$
\left[\ln Z_{u}\right] \mid Z_{t} \sim N\left(\ln \left(Z_{t}\right)+\left(\alpha_{Z}-\frac{1}{2} \sigma_{Z}^{2}\right)(u-t), \sigma_{Z}^{2}(u-t)\right), \quad u>t
$$

Lemma 3 The moments of the variable $Z_{t}$ under the risk-neutral measure are the following:

$$
\begin{aligned}
E\left(Z_{t}\right) & =Z_{0} e^{\alpha_{Z} t} \\
V\left(Z_{t}\right) & =\left[E\left(Z_{t}\right)\right]^{2}\left[e^{\sigma_{Z}^{2} t}-1\right] \\
\operatorname{Cov}\left(Z_{t}, Z_{s}\right) & =Z_{0}^{2} e^{\alpha_{Z}(t+s)}\left[e^{\sigma_{Z}^{2} s}-1\right], s<t
\end{aligned}
$$


Proof: See Appendix.

Obviously, for $s=t$, we obtain

$$
\operatorname{Cov}\left(Z_{t}, Z_{t}\right)=Z_{0}^{2} e^{\alpha_{Z}(t+t)}\left[e^{\sigma_{Z}^{2} t}-1\right]=Z_{0}^{2} e^{2 \alpha_{Z} t}\left[e^{\sigma_{Z}^{2} t}-1\right]=V\left(Z_{t}\right)
$$

The following proposition generalizes the Black-Scholes option pricing formula:

Proposition 1 The price at time 0 of an European call option on $Z$ that matures at time $T$ and with strike price $K$ is given by

$$
C(Z, 0, T, K)=e^{-r T} E\left(Z_{T}\right) N\left(d_{1}\right)-K e^{-r T} N\left(d_{2}\right)
$$

where

$$
\begin{aligned}
d_{1} & =\frac{\ln \left(e^{-\alpha_{Z} T} E\left(Z_{T}\right) / K\right)+\left(\alpha_{Z}+\frac{1}{2} \sigma_{Z}^{2}\right) T}{\sigma_{Z} \sqrt{T}} \\
d_{2} & =d_{1}-\sigma_{Z} \sqrt{T}
\end{aligned}
$$

Proof: See Appendix.

The prices of European put options can be easily obtained using the putcall parity:

$$
P(Z, 0, T, K)=C(Z, 0, T, K)-e^{-r T} E\left(Z_{T}\right)+K e^{-r T}
$$

Let $S_{t}$ denote the stock price at time $t$. We suppose that $S_{t}$ follows the risk-neutral process

$$
d S_{t}=(r-q) S_{t} d t+\sigma S_{t} d W_{t}
$$


where $q$ is the continuous dividend yield of the stock and $\sigma$ is a constant.

The solution for this process is given by

$$
S_{t}=S_{0} \exp \left\{\left(r-q-\frac{1}{2} \sigma^{2}\right) t+\sigma W_{t}\right\}
$$

Note that application of Proposition 1 to the stock price process (11) leads to the Black-Scholes formula adjusted by dividends, as derived by Merton (1973).

\section{Geometric Australian Asian Options}

We consider $n$ monitoring dates so that the time interval $[0, T]$ is partitioned in the following way:

$$
\left\{t_{0}=0<t_{1}<t_{2}<\cdots<t_{n}=T\right\}, t_{i}-t_{i-1}=\frac{T}{n}=\Delta t, \forall i=1, \cdots, n .
$$

Let $S=\left\{S_{t_{i}} \equiv S_{i}, i=0,1, \cdots, n\right\}$ be the price process for the stock. We define the geometric mean of the $n$ stock prices $S_{1}, \cdots, S_{n}$ as

$$
G_{n}=\left(S_{1} \cdots S_{n}\right)^{\frac{1}{n}}=\left(\prod_{i=1}^{n} S_{i}\right)^{\frac{1}{n}}, \quad G_{0} \equiv S_{0}
$$

Using (12), we have

$$
G_{n}=S_{0} \exp \left\{\left(r-q-\frac{1}{2} \sigma^{2}\right) \frac{n+1}{2} \Delta t+\frac{\sigma}{n} \sum_{i=1}^{n} W_{t_{i}}\right\}
$$

Looking at (12) and (13), and using $t_{n}=n \Delta t$, we have

$$
\begin{aligned}
& \frac{S_{n}}{G_{n}}=\exp \left\{\left(r-q-\frac{1}{2} \sigma^{2}\right) \frac{n-1}{2} \Delta t+\frac{\sigma}{n}\left[n W_{t_{n}}-\sum_{i=1}^{n} W_{t_{i}}\right]\right\} \\
& \frac{G_{n}}{S_{n}}=\exp \left\{-\left(r-q-\frac{1}{2} \sigma^{2}\right) \frac{n-1}{2} \Delta t-\frac{\sigma}{n}\left[n W_{t_{n}}-\sum_{i=1}^{n} W_{t_{i}}\right]\right\}
\end{aligned}
$$


It is clear from (13)-(15) that the geometric average and both ratios are lognormally distributed. Consequently, we can apply Proposition 2 to price options on these assets after computing their moments. We now state a Lemma that will be useful to obtain these prices.

Lemma 4 Given the Brownian motions $W_{t_{i}}, i=1, \cdots, n$, it is verified that

$$
\begin{aligned}
V\left(\sum_{i=1}^{n} W_{t_{i}}\right) & =\frac{(n+1)\left(n+\frac{1}{2}\right) n}{3} \Delta t \\
V\left(n W_{t_{n}}-\sum_{i=1}^{n} W_{t_{i}}\right) & =\frac{(n-1)\left(n-\frac{1}{2}\right) n}{3} \Delta t
\end{aligned}
$$

Proof: See Appendix.

Proposition 2 We consider European call options on $S_{n} / G_{n}$ and $G_{n} / S_{n}$ that mature at time $T$ and with strike price $K$. The prices at time 0 of these options are given by expression (9), where the expected value and the logarithmic variance of the asset at maturity are given by the following table: ${ }^{9}$

$$
\begin{array}{c|cc}
Z_{n} & E\left(Z_{n}\right) & \sigma_{Z}^{2} T \\
\hline G_{n} & S_{0} \exp \left\{\left(r-q-\frac{n-1}{6 n} \sigma^{2}\right) \frac{n+1}{2 n} T\right\} & \frac{(n+1)\left(n+\frac{1}{2}\right)}{3 n^{2}} \sigma^{2} T \\
S_{n} / G_{n} & \exp \left\{\left(r-q-\frac{n+1}{6 n} \sigma^{2}\right) \frac{n-1}{2 n} T\right\} & \frac{(n-1)\left(n-\frac{1}{2}\right)}{3 n^{2}} \sigma^{2} T \\
G_{n} / S_{n} & \exp \left\{-\left(r-q-\frac{5 n-1}{6 n} \sigma^{2}\right) \frac{n-1}{2 n} T\right\} & \frac{(n-1)\left(n-\frac{1}{2}\right)}{3 n^{2}} \sigma^{2} T
\end{array}
$$

Proof: See Appendix.

The expression obtained in this Proposition is the Black-Scholes formula with a volatility parameter $\sigma_{Z}$ and a continuous dividend yield $\delta=r-\alpha_{Z_{n}}$ as given by the next table: 


\begin{tabular}{c|c}
$Z_{n}$ & $\delta$ \\
\hline$G_{n}$ & $\left(\frac{n-1}{n+1} r+q+\frac{n-1}{6 n} \sigma^{2}\right) \frac{n+1}{2 n}$ \\
$S_{n} / G_{n}$ & $\left(\frac{n+1}{n-1} r+q+\frac{n+1}{6 n} \sigma^{2}\right) \frac{n-1}{2 n}$ \\
$G_{n} / S_{n}$ & $\left(\frac{3 n-1}{n-1} r-q-\frac{5 n-1}{6 n} \sigma^{2}\right) \frac{n-1}{2 n}$
\end{tabular}

Note that the prices of Australian Asian options do not depend on the current stock price, $S_{0}$.

Obviously, when $Z_{n}=S_{n}$, we obtain the Black-Scholes formula which does not depend on the partition of the time interval. When $Z_{n}=G_{n}$, we get the price derived by Turnbull and Wakeman (1991) and Ritchken et al (1993).

The effect of the number of monitoring dates $(n)$ on the expected value of the asset is not clear, since it depends on the relationship among $r, q$ and $\sigma$. However, the effect of $n$ on the logarithmic variance, $\sigma_{Z}^{2} T$, is quite obvious as can be seen in Figure 1. The parameter values are $\sigma=0.2$ and $T=1$.

\section{[ Insert Figure 1 about here ]}

We observe the following facts:

- The logarithmic variance of the stock price is constant.

- The logarithmic variance of $G_{n}$ is equal to that of the stock price when $n=1$. Then it decreases with $n$, and converges to $\frac{\sigma^{2}}{3} T$, the logarithmic variance of the continuous geometric average, obtained by Kemna and Vorst (1990).

- The logarithmic variances of $S_{n} / G_{n}$ and $G_{n} / S_{n}$ are equal. This is due to the relationship between the variances of a lognormal variable and 
its reciprocal (see expressions (3)-(5)). This variance increases with $n$ and converges to $\frac{\sigma^{2}}{3} T$.

It can also be of interest to study the effect of $\sigma$ and $T$ on option prices. We leave this analysis for the case of continuous-time means.

Tables 1 and 2 show call and put option prices respectively (multiplied by 100) for different cases and monitoring dates. Call prices are computed using Proposition 2. Put prices are obtained applying expression (10). The interest rate is $10 \%$ and the stock dividend yield is $3 \%$. We include the stock price $\left(S_{n}\right)$ and its geometric average $\left(G_{n}\right)$ as underlying assets as a reference. In both cases, we assume that the initial stock price $\left(S_{0}\right)$ is 1 .

\section{[ Insert Tables 1 and 2 about here ]}

We see that the value of the Australian Asian options are relatively similar to those of geometric Asian options.

For one monitoring date and $G_{n}=S_{T}$, we see that options on $G_{n}$ have the same value as those on the stock. Moreover, Australian options are equal to options on the unity, and their values are given by $\exp \{-r T\} \max \{1-K, 0\}$.

Interestingly, option prices do not necessarily increase with the volatility of the stock price $(\sigma)$ either. This is also true for standard geometric Asian options. For example, from Table 1 we have that when $T=0.5, K=0.8$, and $n=1,000$ the call option on $G_{n}$ has a value of 20.548 and 20.538 for $\sigma=0.2$ and 0.4 , respectively. ${ }^{10}$

We see that option prices do not necessarily increase with time to maturity $(T)$. For example, when $\sigma=0.2, K=0.8$, and $n=100$, Table 1 shows that 
the call option on $G_{n} / S_{n}$ has a value of 18.161 and 16.580 for maturities of 0.5 and 1.0 years, respectively.

The tables show that option prices do not change monotonically with $n$. For instance, in Table 1 we have that when $\sigma=0.2, T=0.5$, and $K=1.1$, call options prices on $G_{n}$ are $3.175,0.905$, and 0.737 , when $n=1,10$ and 100, respectively.

The effect of a change in the exercise price $(K)$ is as expected: call prices decrease and put prices increase with $K$.

As additional reference, the Black-Scholes call option prices (dividend yield $=0$ ) in the four cases studied in Table 1 are 24.027, 27.993, 26.081, and 3.743, and the Black-Scholes put option prices corresponding to Table 2 are $3.400,3.753,8.703$, and 8.378, respectively.

We now define the continuous geometric average of the stock price over the interval $[0, T]$ as

$$
G_{T}=\exp \left\{\frac{1}{T} \int_{0}^{T} \ln \left(S_{t}\right) d t\right\}
$$

Using (12), we have

$$
G_{T}=S_{0} \exp \left\{\frac{1}{2}\left(r-q-\frac{1}{2} \sigma^{2}\right) T+\frac{\sigma}{T} \int_{0}^{T} W_{t} d t\right\}
$$

Looking at (12) and (16), we have

$$
\begin{aligned}
& \frac{S_{T}}{G_{T}}=\exp \left\{\frac{1}{2}\left(r-q-\frac{1}{2} \sigma^{2}\right) T+\frac{\sigma}{T}\left[T W_{T}-\int_{0}^{T} W_{t} d t\right]\right\} \\
& \frac{G_{T}}{S_{T}}=\exp \left\{-\frac{1}{2}\left(r-q-\frac{1}{2} \sigma^{2}\right) T-\frac{\sigma}{T}\left[T W_{T}-\int_{0}^{T} W_{t} d t\right]\right\}
\end{aligned}
$$

We now state a Lemma that will be useful to compute the prices of options on these assets. 
Lemma 5 Given the Brownian motions $W_{t}, t \in[0, T]$, it is verified that

$$
V\left(\int_{0}^{T} W_{t} d t\right)=V\left(T W_{T}-\int_{0}^{T} W_{t} d t\right)=\frac{T^{3}}{3}
$$

Proof: See Appendix.

Proposition 3 We consider European call options on $S_{T} / G_{T}$ and $G_{T} / S_{T}$ that mature at time $T$ and with strike price $K$. The prices at time 0 of these options are given by expression (9), where the expected value and the logarithmic variance of the asset at maturity are given by the following table: ${ }^{11}$

\begin{tabular}{c|cc}
$Z_{T}$ & $E\left(Z_{T}\right)$ & $\sigma_{Z}^{2} T$ \\
\hline$S_{T}$ & $S_{0} \exp \{(r-q) T\}$ & $\sigma^{2} T$ \\
$G_{T}$ & $S_{0} \exp \left\{\frac{1}{2}\left(r-q-\frac{1}{6} \sigma^{2}\right) T\right\}$ & $\frac{\sigma^{2}}{3} T$ \\
$S_{T} / G_{T}$ & $\exp \left\{\frac{1}{2}\left(r-q-\frac{1}{6} \sigma^{2}\right) T\right\}$ & $\frac{\sigma^{2}}{3} T$ \\
$G_{T} / S_{T}$ & $\exp \left\{-\frac{1}{2}\left(r-q-\frac{5}{6} \sigma^{2}\right) T\right\}$ & $\frac{\sigma^{2}}{3} T$
\end{tabular}

Proof: See Appendix.

The option pricing formula given in this Proposition corresponds to a continuous dividend yield $\delta=r-\alpha_{Z_{T}}$ as given by the next table:

\begin{tabular}{c|c}
$Z_{T}$ & $\delta$ \\
\hline$S_{T}$ & $q$ \\
$G_{T}, S_{T} / G_{T}$ & $\frac{1}{2}\left(r+q+\frac{1}{6} \sigma^{2}\right)$ \\
$G_{T} / S_{T}$ & $\frac{1}{2}\left(3 r-q-\frac{5}{6} \sigma^{2}\right)$
\end{tabular}

Notice that:

- The logarithmic variances of both ratios are equal to the one derived by Kemna and Vorst (1990) for the continuous geometric average. The intuition for this result is that, with infinite monitoring dates, the volatility of the ratio depends only on the volatility of the average. This value 
increases with $\sigma$ and is one third of the variance in the Black-Scholes formula.

- The expected value of $G_{T}$ is $S_{0}$ times the expected value of $S_{T} / G_{T}$.

- The expected values of $S_{T} / G_{T}$ and $G_{T} / S_{T}$ do not depend on the current stock price, $S_{0}$.

- The expected values of $G_{T}$ and $S_{T} / G_{T}$ are smaller than that of $S_{T}$.

Figure 2 shows $E\left(Z_{T}\right)$ as a function of $\sigma$. The parameter values are $r=10 \%, q=3 \%, T=1$. We assume $S_{0}=1.2$.

\section{[ Insert Figure 2 about here ]}

We observe that the expected values of $G_{T}$ and $S_{T} / G_{T}$ decrease with $\sigma$, while the expected value of $G_{T} / S_{T}$ increases with $\sigma .^{12}$

Since the logarithmic variance of the assets studied increase with $\sigma$, and their expected values also depend on $\sigma$, we have that option prices can decrease with volatility or time to maturity. This surprising result is analyzed next with more detail.

\section{a) Theta}

To study the effect of a change in $T$ on option prices, we compute the corresponding partial derivative. Replacing the expected value and the logarithmic variance of the asset at maturity in (9) as given in Proposition 1 and differentiating with respect to $T$, we get

$$
\frac{\partial C(.)}{\partial T}=e^{-r T}\left[\left(\alpha_{Z_{T}}-r\right) E\left(Z_{T}\right) N\left(d_{1}\right)+K r N\left(d_{2}\right)+\frac{\sigma_{Z}}{2 \sqrt{T}} E\left(Z_{T}\right) N^{\prime}\left(d_{1}\right)\right]
$$


If $q>0$, we have that $\alpha_{Z_{T}}-r<0$ for all the assets except for $G_{T} / S_{T}$. Consequently, the effect of $T$ on call options on these assets is undetermined. If $q=0$, we have that $\alpha_{s_{T}}-r=0$, and the stock call price increases with $T$. For the ratio $G_{T} / S_{T}$, we have that $\alpha_{Z_{T}}-r>0 \Leftrightarrow r<\frac{1}{3}\left(q+\frac{5}{6} \sigma^{2}\right)$. In this case, an increase in $T$ leads to a higher call option price.

To analyze the effect of an increase of $T$ on the put price, we use the put-call parity (10) and the derivative obtained in (19) to get

$$
\frac{\partial P(.)}{\partial T}=e^{-r T}\left[\left(r-\alpha_{Z_{T}}\right) E\left(Z_{T}\right) N\left(-d_{1}\right)-K r N\left(-d_{2}\right)+\frac{\sigma_{Z}}{2 \sqrt{T}} E\left(Z_{T}\right) N^{\prime}\left(d_{1}\right)\right]
$$

The sign of this derivative can be positive or negative, depending on the parameter values. For all the assets except $G_{T} / S_{T}$, we have $r-\alpha_{Z_{T}} \geq 0$. Then, for these assets, the effect of $T$ on the put price depends on how large is the second term into brackets. If the exercise price is low enough, the put price will increase with $T$, while for large strikes the opposite will take place. For the ratio $G_{T} / S_{T}$, we have that $r-\alpha_{Z_{T}}>0 \Leftrightarrow r>\frac{1}{3}\left(q+\frac{5}{6} \sigma^{2}\right)$. In this case, an increase in $T$ can lead to a higher put price if the exercise price is small.

Figure 3 plots geometric Australian option prices as a function of time to maturity. The averages are computed with infinite monitoring dates. The parameters are: $r=0.1, q=0.03, \sigma=0.2, K=0.8$ for calls and $K=1.2$ for puts.

\section{[ Insert Figure 3 about here ]}

We see that, in this case, the price of a call option on $S_{T} / G_{T}$ increases with $T$. However, the price of a call option on $G_{T} / S_{T}$ decreases with $T$. The 
latter result is due to the fact that $r>\frac{1}{3}\left(q+\frac{5}{6} \sigma^{2}\right)$, so that $\alpha_{Z_{T}}-r<0$ and $\partial C(.) / \partial T$ can be negative.

Since the exercise price for the put options is relatively high $(K=1.2)$, we see that the price of the put option on $S_{T} / G_{T}$ decreases with $T$. The same occurs for a put option on $G_{T} / S_{T}$ when time to maturity is small (between 0 and 0.65 years). For higher $T$, the put price increases. When $T>1.5$, the put price decreases again. Interestingly, if we reduce the exercise price to $K=1.1$, the put price increases for all $T$.

\section{b) Vega}

Replacing the expected value and the logarithmic variance of the asset at maturity in (9) as given in Proposition 1 and differentiating the resulting expression with respect to $\sigma$, we obtain that the effect of a change in $\sigma$ on the price of a call option is given by

$$
\nu_{C}=\frac{\partial C(.)}{\partial \sigma}=e^{-r T} E\left(Z_{T}\right) \sqrt{T}\left[\frac{\partial \alpha_{Z}}{\partial \sigma} \sqrt{T} N\left(d_{1}\right)+\frac{\partial \sigma_{Z}}{\partial \sigma} N^{\prime}\left(d_{1}\right)\right]
$$

The partial derivatives into brackets are:

\begin{tabular}{c|cc}
$Z_{T}$ & $\frac{\partial \alpha_{Z}}{\partial \sigma}$ & $\frac{\partial \sigma_{Z}}{\partial \sigma}$ \\
\hline$S_{T}$ & 0 & 1 \\
$G_{T}$ & $-\frac{1}{6} \sigma$ & $\frac{1}{\sqrt{3}}$ \\
$S_{T} / G_{T}$ & $-\frac{1}{6} \sigma$ & $\frac{1}{\sqrt{3}}$ \\
$G_{T} / S_{T}$ & $\frac{5}{6} \sigma$ & $\frac{1}{\sqrt{3}}$
\end{tabular}

Therefore, we obtain

$$
\nu_{C}\left(S_{T}\right)=\frac{\partial C(.)}{\partial \sigma}=S_{0} \sqrt{T} N^{\prime}\left(d_{1}\right)>0
$$




$$
\begin{aligned}
\nu_{C}\left(G_{T}\right) & =\frac{\partial C(.)}{\partial \sigma}=e^{-r T} E\left(G_{T}\right) \sqrt{T}\left[-\frac{1}{6} \sigma \sqrt{T} N\left(d_{1}\right)+\frac{1}{\sqrt{3}} N^{\prime}\left(d_{1}\right)\right] \\
\nu_{C}\left(S_{T} / G_{T}\right) & =\frac{\partial C(.)}{\partial \sigma}=e^{-r T} E\left(S_{T} / G_{T}\right) \sqrt{T}\left[-\frac{1}{6} \sigma \sqrt{T} N\left(d_{1}\right)+\frac{1}{\sqrt{3}} N^{\prime}\left(d_{1}\right)\right] \\
\nu_{C}\left(G_{T} / S_{T}\right) & =\frac{\partial C(.)}{\partial \sigma}=e^{-r T} E\left(G_{T} / S_{T}\right) \sqrt{T}\left[\frac{5}{6} \sigma \sqrt{T} N\left(d_{1}\right)+\frac{1}{\sqrt{3}} N^{\prime}\left(d_{1}\right)\right]>0
\end{aligned}
$$

It is straightforward to show that

$$
\begin{aligned}
\nu_{C}\left(G_{T}\right) & <0 \Leftrightarrow \frac{N^{\prime}\left(d_{1}\right)}{\sigma N\left(d_{1}\right)}<\frac{1}{2} \sqrt{\frac{T}{3}} \\
\nu_{C}\left(S_{T} / G_{T}\right) & <0 \Leftrightarrow \frac{N^{\prime}\left(d_{1}\right)}{\sigma N\left(d_{1}\right)}<\frac{1}{2} \sqrt{\frac{T}{3}}
\end{aligned}
$$

Hence, the vega of a call option on in the Kemna and Vorst (1990) model and the vega of a geometric Australian asian call option on $S_{T} / G_{T}$ can be negative. As shown later, this can occur for reasonable parameter values.

To analyze the effect of an increase of $\sigma$ on the put price, we use the put-call parity (10) and the derivative obtained in (21) to obtain

$$
\nu_{P}=\frac{\partial P(.)}{\partial \sigma}=e^{-r T} E\left(Z_{T}\right) \sqrt{T}\left[\frac{\partial \sigma_{Z}}{\partial \sigma} N^{\prime}\left(d_{1}\right)-\frac{\partial \alpha_{Z}}{\partial \sigma} \sqrt{T} N\left(-d_{1}\right)\right]
$$

Therefore, the vega of put options on our assets are:

$$
\begin{aligned}
\nu_{P}\left(S_{T}\right) & =\frac{\partial P(.)}{\partial \sigma}=S_{0} \sqrt{T} N^{\prime}\left(d_{1}\right)>0 \\
\nu_{P}\left(G_{T}\right) & =\frac{\partial P(.)}{\partial \sigma}=e^{-r T} E\left(G_{T}\right) \sqrt{T}\left[\frac{1}{6} \sigma \sqrt{T} N\left(-d_{1}\right)+\frac{1}{\sqrt{3}} N^{\prime}\left(d_{1}\right)\right]>0 \\
\nu_{P}\left(S_{T} / G_{T}\right) & =\frac{\partial P(.)}{\partial \sigma}=e^{-r T} E\left(S_{T} / G_{T}\right) \sqrt{T}\left[\frac{1}{6} \sigma \sqrt{T} N\left(-d_{1}\right)+\frac{1}{\sqrt{3}} N^{\prime}\left(d_{1}\right)\right]>0 \\
\nu_{P}\left(G_{T} / S_{T}\right) & =\frac{\partial P(.)}{\partial \sigma}=e^{-r T} E\left(G_{T} / S_{T}\right) \sqrt{T}\left[-\frac{5}{6} \sigma \sqrt{T} N\left(-d_{1}\right)+\frac{1}{\sqrt{3}} N^{\prime}\left(d_{1}\right)\right]
\end{aligned}
$$

It is clear that

$$
\nu_{P}\left(G_{T} / S_{T}\right)<0 \Leftrightarrow \frac{N^{\prime}\left(d_{1}\right)}{\sigma N\left(-d_{1}\right)}<\frac{5}{2} \sqrt{\frac{T}{3}}
$$


As before, this inequality can hold for reasonable parameter values.

Figure 4 exhibits geometric Australian option prices as a function of volatility $(\sigma)$. The averages are computed with infinite monitoring dates. The parameters are: $r=0.1, q=0.03, T=0.1, K=0.8$ for calls and $K=1.1$ for puts.

\section{[ Insert Figure 4 about here ]}

We see that the price of the call option on $S_{T} / G_{T}$ first decreases and then increases with volatility. The vega of this option is zero when $\sigma=0.67$. As expected, the price of the call option on $G_{T} / S_{T}$ and a put option on $S_{T} / G_{T}$ always increase with $\sigma$. However, the price of the put option on $G_{T} / S_{T}$ first decreases and then increases with volatility. The vega of this option is zero when sigma $=0.36$.

To summarize the results, both call and put geometric Australian option prices can increase and decrease with either time to maturity or volatility. This is clearly seen in Figure 5, that shows option prices as a function of both variables. The parameter values are: $r=0.1, q=0.03, n=\infty, K=0.8$ for the call option and $K=1.1$ for the put option.

\section{[ Insert Figure 5 about here ]}

Finally, Tables 1 and 2 present geometric option prices when $n=\infty$. Note that the option on $S_{n} / G_{n}$ is equivalent to the option on $G_{n}$. This happens because we are taking $S_{0}=1$. We see that option prices for continuous averages are almost identical to those for discrete averages with 1,000 monitoring dates. For example, from Table 2 we have that when $\sigma=0.2, T=1, K=1.0$ 
and $n=\infty$, the put options on $G_{n}, S_{n} / G_{n}$, and $G_{n} / S_{n}$ have values of 2.935, 2.935, and 5.002, respectively, while that when $n=1,000$ those prices are $2.937,2.933$, and 5.000, respectively.

\section{Arithmetic Australian Asian Options}

We define the discrete arithmetic mean of the $n$ stock prices $S_{1}, \cdots, S_{n}$ as

$$
A_{n}=\frac{1}{n}\left(S_{1}+\cdots+S_{n}\right)=\frac{1}{n} \sum_{i=1}^{n} S_{i}, \quad A_{0} \equiv S_{0}
$$

The continuous counterpart is given by

$$
A_{T}=\frac{1}{T} \int_{0}^{T} S_{t} d t
$$

As mentioned previously, the distribution of $A_{n}$ is unknown. Therefore, we can not apply Proposition 1 to price options. As described in the following sections, two ways to overcome this problem are:

- To approximate the true distribution with an alternative one.

- To approximate the distribution of $A_{n}$ with that of $A_{T}$.

\subsection{Pricing the Options with the Edgeworth / Wilkin- son Approximation}

To price options, we approximate the risk-neutral distribution of the underlying asset at maturity with a tractable distribution. We perform this approximation by expanding the true distribution around the approximating one. This approach is called generalized Edgeworth series expansion. The 
coefficients of this expansion are function of the moments of the true and approximating distribution. Considering up to four terms in this expansion and specifying the approximating distribution to be lognormal, we will show that the (approximate) option price is equal to the Black-Scholes price plus three adjustment terms. These terms depend, respectively, on the difference between the variance, skewness, and kurtosis of the true and the lognormal distribution. The intuition is that the first four moments of the distribution are enough to reflect the effects of the distribution on option prices.

More concretely, we approximate the true probability distribution, $F(s)$, with an approximating distribution, $A(s)$. It is assumed that both distributions have continuous density functions, $f(s)$ and $a(s)$. We employ the following notation:

$$
\begin{aligned}
\alpha_{j}(F) & =\int_{-\infty}^{\infty} s^{j} f(s) d s \\
\mu_{j}(F) & =\int_{-\infty}^{\infty}\left(s-\alpha_{1}(F)\right)^{j} f(s) d s \\
\Psi(F, t) & =\int_{-\infty}^{\infty} e^{i t s} f(s) d s, \quad i=\sqrt{-1}
\end{aligned}
$$

where $\alpha_{j}(F)$ and $\mu_{j}(F)$ are, respectively, the j-th non-central and central moments of $F$ and $\Psi(F, t)$ is the characteristic function of $F{ }^{13}$

Following Stuart and Ord (1987), the cumulants $k_{j}(F)$ of the distribution $F$ are defined by the identity in $t$

$$
\exp \left(\sum_{j=1}^{\infty} k_{j}(F) \frac{t^{j}}{j !}\right)=\sum_{j=0}^{\infty} \alpha_{j}(F) \frac{t^{j}}{j !}
$$

or, equivalently

$$
\ln \Psi(F, t)=\sum_{j=1}^{\infty} k_{j}(F) \frac{(i t)^{j}}{j !}
$$


For practical purposes, we only need the first four cumulants in the Edgeworth series expansion. These cumulants are, respectively, the mean, the variance, the coefficient of skewness and the excess of kurtosis:

$$
\begin{array}{ll}
k_{1}(F)=\alpha_{1}(F), & k_{2}(F)=\mu_{2}(F) \\
k_{3}(F)=\mu_{3}(F), & k_{4}(F)=\mu_{4}(F)-3 \mu_{2}^{2}(F)
\end{array}
$$

Jarrow and Rudd (1982) prove the following series expansion for $f(s)$ around $a(s)$ :

$$
\begin{aligned}
f(s)= & a(s)+\frac{k_{2}(F)-k_{2}(A)}{2 !} \frac{d^{2} a(s)}{d s^{2}}-\frac{k_{3}(F)-k_{3}(A)}{3 !} \frac{d^{3} a(s)}{d s^{3}} \\
& +\frac{k_{4}(F)-k_{4}(A)+3\left(k_{2}(F)-k_{2}(A)\right)^{2}}{4 !} \frac{d^{4} a(s)}{d s^{4}}+\varepsilon(s)
\end{aligned}
$$

where, by construction, $k_{1}(F)$ is set equal to $k_{1}(A)$.

The difference between $f(s)$ and $a(s)$ depends on the cumulants of both distributions with weighting factors given by the derivatives of $a(s)$. The terms on the right-hand side of (25) reflect any difference in variance, skewness and kurtosis and variance between $f(s)$ and $a(s)$. The residual error, $\varepsilon(s)$, includes any remaining difference.

Now, we employ (25) to obtain an approximate option price. Using $f(s)$ as the true distribution of the asset price at maturity, we obtain the expected value at maturity of an option on this asset. Then, this expansion provides an approximated expected value for the option at maturity in terms of the approximating distribution, $a(s)$.

In a risk-neutral world, the true price of the call option, $C(F)$, is obtained by discounting its expected value at the risk-free rate:

$$
C(F)=e^{-r T} \int_{-\infty}^{\infty} \max \left\{S_{T}-K, 0\right\} d F\left(S_{T}\right)
$$


Using (25) and a little algebra, this price becomes

$$
\begin{aligned}
C(F)= & C(A)+e^{-r T} \frac{k_{2}(F)-k_{2}(A)}{2 !} a(K)-\left.e^{-r T} \frac{k_{3}(F)-k_{3}(A)}{3 !} \frac{d a}{d S_{T}}\right|_{K} \\
& +\left.e^{-r T} \frac{k_{4}(F)-k_{4}(A)+3\left(k_{2}(F)-k_{2}(A)\right)^{2}}{4 !} \frac{d^{2} a}{d S_{T}^{2}}\right|_{K}+\varepsilon(K)
\end{aligned}
$$

where

$$
C(A)=e^{-r T} \int_{-\infty}^{\infty} \max \left\{S_{T}-K, 0\right\} d A\left(S_{T}\right)
$$

A natural candidate for the approximating distribution is the lognormal one. In this case, $C(A)$ is equal to the Black-Scholes option price.

Equation (26) shows that the true option price is equal to $C(A)$ plus three adjustment terms. As before, these terms correct for difference in variance, skewness and kurtosis and variance between the distributions $F$ and $A$ while the term $\varepsilon(K)$ includes the residual error.

As mentioned in the Introduction, the Wilkinson approximation is a particular case of the Edgeworth expansion, where just the first two cumulants are used.

\subsection{Pricing the Options with the Gamma Distribution}

It is known that the infinite sum of lognormal distributions is a reciprocal gamma distribution. Using this distribution as state-price density function, Milevsky and Posner (1998) obtain a closed-form expression for the price of arithmetic Asians options. The solution is the same as the Black-Scholes formula where the normal distribution is replaced by the gamma one.

We briefly summarize the main characteristics of the gamma distribution. Let $X$ be gamma distributed with parameters $\alpha$ and $\beta$, that is, $X \sim \Gamma(\alpha, \beta)$. 
Its density function is given by

$$
g(x)=\frac{\beta^{-\alpha} x^{\alpha-1} \exp \left\{-\frac{x}{\beta}\right\}}{\Gamma(\alpha)}, x>0
$$

where $\Gamma(x)$ is the gamma function, defined as

$$
\Gamma(x)=\int_{0}^{\infty} t^{x-1} e^{-t} d t
$$

The mean and variance of the gamma distribution are

$$
E(X)=\alpha \beta, \quad V(X)=\alpha \beta^{2} .
$$

If we define $Y=\frac{1}{X}$, then $Y$ follows a reciprocal gamma distribution. Its first two non-central moments are

$$
\begin{aligned}
& M_{1}=E(Y)=\frac{1}{\beta(\alpha-1)} \\
& M_{2}=E\left(Y^{2}\right)=\frac{1}{\beta^{2}(\alpha-1)(\alpha-2)}
\end{aligned}
$$

The variance is given by

$$
V(Y)=M_{2}-M_{1}^{2}=\frac{1}{\beta^{2}(\alpha-1)^{2}(\alpha-2)}
$$

It is straightforward to obtain the following relationships:

$$
\alpha=\frac{2 M_{2}-M_{1}^{2}}{M_{2}-M_{1}^{2}}, \quad \beta=\frac{M_{2}-M_{1}^{2}}{M_{1} M_{2}}
$$

Hence, to price option, we must obtain the first two risk-neutral moments $\left(M_{1}, M_{2}\right)$ of the underlying asset at maturity. Then, we compute $\alpha$ and $\beta$ using (27). Finally, we use the cumulative density function of the gamma distribution as $N($.$) in the Black-Scholes formula$

In the next section, we compute the moments of the arithmetic mean and the ratios to price options on these assets with the methods previously described. 


\subsection{Computation of Moments}

The following Lemma summarizes several properties of the stock price that will be used later:

Lemma 6 For $i, j=1,2, \cdots, n$ and $k \in \mathbb{R}$, we have

$$
\begin{aligned}
E\left(S_{i}^{k}\right) & =S_{0}^{k} \exp \left\{k\left(r-q+\frac{k-1}{2} \sigma^{2}\right) i \Delta t\right\} \\
E\left(S_{i} S_{j}^{k}\right) & =E\left(S_{i}\right) E\left(S_{j}^{k}\right) \exp \left\{k \sigma^{2} \min \{i, j\} \Delta t\right\} \\
E\left(S_{i} S_{j} S_{n}^{k}\right) & =E\left(S_{i} S_{j}\right) E\left(S_{n}^{k}\right) \exp \left\{k \sigma^{2}(i+j) \Delta t\right\}
\end{aligned}
$$

Moreover, for $k \in \mathbb{R}$, we have

$$
\begin{aligned}
& E\left(A_{n} S_{n}^{k}\right)=\frac{S_{0}}{n} E\left(S_{n}^{k}\right) h_{1}\left(r^{*}\right) \\
& E\left(A_{n}^{2} S_{n}^{k}\right)=\left(\frac{S_{0}}{n}\right)^{2} E\left(S_{n}^{k}\right)\left[2 f_{1}\left(r^{*}+\sigma^{2}\right)\left(h_{1}\left(2 r^{*}+\sigma^{2}\right)-h_{1}\left(r^{*}\right)\right)-h_{1}\left(2 r^{*}+\sigma^{2}\right)\right]
\end{aligned}
$$

where

$$
\begin{aligned}
h_{1}(x) & =\sum_{i=1}^{n} e^{x i \Delta t}=f_{1}(x)\left(e^{x n \Delta t}-1\right), x \neq 0, \quad h_{1}(0)=n \\
f_{1}(x) & =\frac{e^{x \Delta t}}{e^{x \Delta t}-1}, x \neq 0 \\
r^{*} & =r-q+k \sigma^{2}
\end{aligned}
$$

Proof: See Appendix.

The following Lemma gives the moments of $A_{n}, S_{n} / A_{n}$ and $A_{n} / S_{n}$ : 


\section{Lemma 7}

1. The moments of the variable $A_{n}$ are given by

$$
\begin{aligned}
E\left(A_{n}\right)= & \frac{S_{0}}{n} h_{1}(r-q) \\
\operatorname{Cov}\left(A_{n}, S_{n}\right)= & \frac{S_{0}}{n} E\left(S_{n}\right)\left[h_{1}\left(r-q+\sigma^{2}\right)-h_{1}(r-q)\right] \\
V\left(A_{n}\right)= & \left(\frac{S_{0}}{n}\right)^{2}\left[2 f_{1}\left(r-q+\sigma^{2}\right)\left(h_{1}\left(2(r-q)+\sigma^{2}\right)-h_{1}(r-q)\right)\right. \\
& \left.-h_{1}\left(2(r-q)+\sigma^{2}\right)-\left(h_{1}(r-q)\right)^{2}\right]
\end{aligned}
$$

2. The moments of the variable $S_{n} / A_{n}, n \geq 2$ can be approximated by

$$
\begin{aligned}
& E\left(\frac{S_{n}}{A_{n}}\right) \simeq \frac{E\left(S_{n}\right)}{E\left(A_{n}\right)}-\frac{1}{\left(E\left(A_{n}\right)\right)^{2}} \operatorname{Cov}\left(A_{n}, S_{n}\right)+\frac{E\left(S_{n}\right)}{\left(E\left(A_{n}\right)\right)^{3}} V\left(A_{n}\right) \\
& V\left(\frac{S_{n}}{A_{n}}\right) \simeq\left(\frac{E\left(S_{n}\right)}{E\left(A_{n}\right)}\right)^{2}\left(\frac{V\left(S_{n}\right)}{\left(E\left(S_{n}\right)\right)^{2}}+\frac{V\left(A_{n}\right)}{\left(E\left(A_{n}\right)\right)^{2}}-2 \frac{\operatorname{Cov}\left(A_{n}, S_{n}\right)}{E\left(S_{n}\right) E\left(A_{n}\right)}\right)
\end{aligned}
$$

with $E\left(A_{n}\right), \operatorname{Cov}\left(A_{n}, S_{n}\right)$ and $V\left(A_{n}\right)$ as given by (36)-(38).

3. The moments of the variable $A_{n} / S_{n}, n \geq 2$ are given by

$$
\begin{aligned}
E\left(\frac{A_{n}}{S_{n}}\right)= & \frac{1}{n} \exp \left\{-n\left(r-q-\sigma^{2}\right) \Delta t\right\} h_{1}\left(r-q-\sigma^{2}\right) \\
V\left(\frac{A_{n}}{S_{n}}\right)= & \left(\frac{1}{n}\right)^{2} \exp \left\{-n\left(2(r-q)-3 \sigma^{2}\right) \Delta t\right\} \\
& \times\left[2 f_{1}\left(r-q-\sigma^{2}\right)\left(h_{1}\left(2(r-q)-3 \sigma^{2}\right)-h_{1}\left(r-q-2 \sigma^{2}\right)\right)\right. \\
& \left.-h_{1}\left(2(r-q)-3 \sigma^{2}\right)-\exp \left\{-n \sigma^{2} \Delta t\right\} h_{1}^{2}\left(r-q-\sigma^{2}\right)\right](40)
\end{aligned}
$$

with $h_{1}($.$) and f_{1}($.$) as given by (33) and (34), respectively.$

Proof: See Appendix. 
Remark 1 Several particular cases can be highlighted:

1. If $r=q-\sigma^{2}$, the moments of the variable $A_{n}$ are given by

$$
\begin{aligned}
E\left(A_{n}\right) & =\frac{S_{0}}{n} h_{1}\left(-\sigma^{2}\right) \\
\operatorname{Cov}\left(A_{n}, S_{n}\right) & =\frac{S_{0}}{n} E\left(S_{n}\right)\left(n-h_{1}\left(-\sigma^{2}\right)\right) \\
V\left(A_{n}\right) & =2\left(\frac{S_{0}}{n}\right)^{2} f_{1}\left(\sigma^{2}\right) e^{-(n+1) \sigma^{2} \Delta t} \sum_{i=1}^{n}\left(\cosh \left(\sigma^{2} i \Delta t\right)-1\right)
\end{aligned}
$$

2. If $r=q+\sigma^{2}$, the moments of the variable $A_{n} / S_{n}, n \geq 2$ are given by

$$
\begin{aligned}
& E\left(\frac{A_{n}}{S_{n}}\right)=1 \\
& V\left(\frac{A_{n}}{S_{n}}\right)=\left(\frac{1}{n}\right)^{2}\left[\left(2 f_{1}\left(\sigma^{2}\right)-1\right)\left(e^{-\sigma^{2} \Delta t} h_{1}\left(\sigma^{2}\right)-n\right)-n(n-1)\right]
\end{aligned}
$$

Proof: See Appendix.

The following Lemma gives the moments of $A_{T}, S_{T} / A_{T}$ and $A_{T} / S_{T}$ :

Lemma 8 For $k \in \mathbb{R}$, we have

$$
\begin{aligned}
& E\left(A_{T} S_{T}^{k}\right)=\frac{S_{0}}{T} E\left(S_{T}^{k}\right) \Phi\left(r^{*}\right) \\
& E\left(A_{T}^{2} S_{T}^{k}\right)=2\left(\frac{S_{0}}{T}\right)^{2} E\left(S_{T}^{k}\right) \frac{\Phi\left(2 r^{*}+\sigma^{2}\right)-\Phi\left(r^{*}\right)}{r^{*}+\sigma^{2}}
\end{aligned}
$$

with

$$
\Phi(x)=\frac{\exp \{x T\}-1}{x}, x \neq 0, \quad \Phi(0)=T
$$

and $r^{*}$ as given by (35).

Proof: See Appendix. 


\section{Lemma 9}

1. The moments of the variable $A_{T}$ are given by

$$
\begin{aligned}
E\left(A_{T}\right) & =\frac{S_{0}}{T} \Phi(r-q) \\
\operatorname{Cov}\left(A_{T}, S_{T}\right) & =\frac{S_{0}}{T} E\left(S_{T}\right)\left[\Phi\left(r-q+\sigma^{2}\right)-\Phi(r-q)\right] \\
V\left(A_{T}\right) & =\left(\frac{S_{0}}{T}\right)^{2}\left[2 \frac{\Phi\left(2(r-q)+\sigma^{2}\right)-\Phi(r-q)}{r-q+\sigma^{2}}-(\Phi(r-q))^{2}\right]
\end{aligned}
$$

2. The moments of the variable $S_{T} / A_{T}$ can be approximated by

$$
\begin{aligned}
& E\left(\frac{S_{T}}{A_{T}}\right) \simeq \frac{E\left(S_{T}\right)}{E\left(A_{T}\right)}-\frac{1}{\left(E\left(A_{T}\right)\right)^{2}} \operatorname{Cov}\left(A_{T}, S_{T}\right)+\frac{E\left(S_{T}\right)}{\left(E\left(A_{T}\right)\right)^{3}} V\left(A_{T}\right) \\
& V\left(\frac{S_{T}}{A_{T}}\right) \simeq\left(\frac{E\left(S_{T}\right)}{E\left(A_{T}\right)}\right)^{2}\left(\frac{V\left(S_{T}\right)}{\left(E\left(S_{T}\right)\right)^{2}}+\frac{V\left(A_{T}\right)}{\left(E\left(A_{T}\right)\right)^{2}}-2 \frac{\operatorname{Cov}\left(A_{T}, S_{T}\right)}{E\left(S_{T}\right) E\left(A_{T}\right)}\right)
\end{aligned}
$$

with $E\left(A_{T}\right), \operatorname{Cov}\left(A_{T}, S_{T}\right)$ and $V\left(A_{T}\right)$ as given by (44)-(46).

3. The moments of the variable $A_{T} / S_{T}$ are given by

$$
\begin{aligned}
E\left(\frac{A_{T}}{S_{T}}\right)= & \frac{1}{T} \Phi\left(\sigma^{2}-(r-q)\right) \\
V\left(\frac{A_{T}}{S_{T}}\right)= & \left(\frac{1}{T}\right)^{2} \exp \left\{-\left(2(r-q)-3 \sigma^{2}\right) T\right\} \\
& \times\left[2 \frac{\Phi\left(2(r-q)-3 \sigma^{2}\right)-\Phi\left(r-q-2 \sigma^{2}\right)}{r-q-\sigma^{2}}\right. \\
& \left.-\exp \left\{-\sigma^{2} T\right\} \Phi^{2}\left(r-q-\sigma^{2}\right)\right]
\end{aligned}
$$

Proof: See Appendix. 
Remark 2 Several particular cases can be highlighted:

1. If $r=q-\sigma^{2}$, the moments of the variable $A_{T}$ are given by

$$
\begin{aligned}
E\left(A_{T}\right) & =\frac{S_{0}}{T} \Phi\left(-\sigma^{2}\right) \\
\operatorname{Cov}\left(A_{T}, S_{T}\right) & =\frac{S_{0}}{T} E\left(S_{T}\right)\left[T-\Phi\left(-\sigma^{2}\right)\right] \\
V\left(A_{T}\right) & =2\left(\frac{S_{0}}{T}\right)^{2} \frac{e^{-\sigma^{2} T}}{\sigma^{4}}\left(\sinh \left(\sigma^{2} T\right)-\sigma^{2} T\right)
\end{aligned}
$$

2. If $r=q+\sigma^{2}$, the moments of the variable $A_{T} / S_{T}$ are given by

$$
\begin{aligned}
E\left(\frac{A_{T}}{S_{T}}\right) & =1 \\
V\left(\frac{A_{T}}{S_{T}}\right) & =\left(\frac{1}{T}\right)^{2}\left[2 \frac{\Phi\left(\sigma^{2}\right)-T}{\sigma^{2}}-T^{2}\right]
\end{aligned}
$$

with $\Phi($.$) as given by (43).$

Proof: See Appendix.

Tables 3 and 4 show arithmetic call and put option prices (multiplied by 100) for different monitoring dates. The interest rate is $10 \%$ and the stock dividend yield is $3 \%$. We price options on $A_{n}, S_{n} / A_{n}$ and $A_{n} / S_{n}$ with three methods: Monte Carlo simulation, ${ }^{14}$ Wilkinson approximation, and gamma distribution.

\section{[ Insert Tables 3 and 4 about here ]}

In the tables, we see that derivative prices with the three methods are very close. For example, in Table 3 we have that when $\sigma=0.20, T=0.5, K=0.8$, and $n=1,000$, the values of call options on $A_{n} / S_{n}$ are 18.319, 18.324, and 18.321, respectively. Thus, Edgeworth expansions do not seem to be needed. 
To price options on $S_{n} / A_{n}$ with both the Wilkinson approximation and the gamma distribution, we have computed its moments using the approximation of Mood et al (1974). In the tables we see that those prices are very similar to the ones obtained with Monte Carlo, so that the approximations seem to work pretty well. For example, in Table 3 we see that when $\sigma=0.2, T=0.5, K=0.8$, and $n=1,000$, the values of call options using the Wilkinson approximation and the gamma distribution are 20.375 and 20.374, respectively, while the value obtained with Monte Carlo simulation is 20.377.

When the average is computed in continuous time (number of monitoring dates $=\infty$ ) we cannot use Monte Carlo simulation. However, as mentioned before, using 1,000 monitoring dates produces option prices very similar to those using continuous average. In Table 4 we see that the values of put options on $S_{n} / A_{n}$ using the Wilkinson approximation and the gamma distribution are 8.863 and 8.887 , respectively, for both 1,000 and $\infty$ monitoring dates.

To understand better why the three method produce very similar results, we plot the risk-neutral probability density function of the arithmetic stock price average in Figure 6.

\section{[ Insert Figure 6 about here ]}

The parameter values are: $r=0.1, q=0, \sigma=0.2, T=1, S_{0}=100$ and $n=\infty$. The expected value of the average price is 105.17 , and the variance 152.74. For $n=\infty$ the true density function is reciprocal gamma, with parameters $\alpha=74.42$ and $\beta=1.29 \mathrm{E}-4$. This function is approximated with a lognormal distribution with the same moments. The density function is 
also estimated with Monte Carlo simulation, using a set of 50 runs of 10,000 paths with 1,000 time steps. We see that, for the parameter values used, the density functions are remarkably similar, hence the price of options on arithmetic stock prices must be close.

\section{Conclusions}

Australian Asian options are options on the ratio of the stock price to its average or viceversa. They show up in variable purchase options, recently studied by Handley (2000).

If the stock price follows a geometric Brownian motion and the average is defined on geometric basis, these ratios also follow a geometric Brownian motion. Thus, we are able to obtain closed-form expressions for the price of the options. However, when the average is defined on arithmetic basis, the risk-neutral distributions of these ratios at maturity are unknown. Hence, to price the options we use a particular case of Edgeworth expansion (known as Wilkinson approximation) as well as a gamma approximation (following Milevsky and Posner (1998)). We compare the results with those obtained with Monte Carlo simulations, and we find that option prices are very similar in the three cases. 


\section{Appendix}

\section{Proof of Lemma 1}

1. For proving expressions (2)-(3), see Johnson and Kotz (1970), p. 115.

As $X^{-1}=e^{-Y}$, application of (2)-(3) gives

$$
\begin{aligned}
E\left(X^{-1}\right) & =\exp \left\{-m+\frac{1}{2} s^{2}\right\}=e^{-2 m} E(X) \\
V\left(X^{-1}\right) & =\left[E\left(X^{-1}\right)\right]^{2}\left[e^{s^{2}}-1\right]
\end{aligned}
$$

2. Consider the variable

$$
\tilde{X}=\left\{\begin{array}{cc}
X & \text { if } \quad X \geq K \\
0 & \text { if } \quad X<K
\end{array}, \quad K \in \mathbb{R}^{+}\right.
$$

Using (1), the expectation of this variable is

$$
E(\tilde{X})=\int_{K}^{\infty} \frac{1}{s \sqrt{2 \pi}} \exp \left\{-\frac{1}{2}\left(\frac{\ln x-m}{s}\right)^{2}\right\} d x
$$

Defining the variable

$$
y=\frac{\ln x-m}{s}
$$

a little algebra leads to

$$
E(\tilde{X})=E(X) N(s-D)
$$

where $N($.) denotes the distribution function of a standard normal variable and $D=\frac{\ln K-m}{s}$. 


\section{Proof of Lemma 3}

The expressions for the mean and variance follow from straightforward application of (2)-(3) in Lemma 1 to $S_{t}$ as given by (12).

To obtain $\operatorname{Cov}\left(Z_{t}, Z_{s}\right)$, we assume $s<t$. We first compute $E\left(Z_{t} Z_{s}\right)$ :

$$
\begin{aligned}
E\left(Z_{t} Z_{s}\right) & =Z_{0}^{2} E\left(\exp \left\{\left(\alpha_{Z}-\frac{1}{2} \sigma_{Z}^{2}\right)(t+s)+\sigma_{Z}\left(W_{t}+W_{s}\right)\right\}\right) \\
& =Z_{0}^{2} \exp \left\{\left(\alpha_{Z}-\frac{1}{2} \sigma_{Z}^{2}\right)(t+s)+\frac{1}{2} \sigma_{Z}^{2} V\left(W_{t}+W_{s}\right)\right\} \\
& =Z_{0}^{2} \exp \left\{\left(\alpha_{Z}-\frac{1}{2} \sigma_{Z}^{2}\right)(t+s)+\frac{1}{2} \sigma_{Z}^{2}(t+s+2 s)\right\} \\
& =Z_{0}^{2} \exp \left\{\alpha_{Z}(t+s)+\sigma_{Z}^{2} s\right\}
\end{aligned}
$$

where we have used (2) in Lemma 1. Then,

$$
\begin{aligned}
\operatorname{Cov}\left(Z_{t}, Z_{s}\right) & =E\left(Z_{t} Z_{s}\right)-E\left(Z_{t}\right) E\left(Z_{s}\right)=Z_{0}^{2} e^{\alpha_{Z}(t+s)+\sigma_{Z}^{2} s}-\left(Z_{0} e^{\alpha_{Z} t}\right)\left(Z_{0} e^{\alpha_{Z} s}\right) \\
& =Z_{0}^{2} e^{\alpha_{Z}(t+s)}\left[e^{\sigma_{Z}^{2} s}-1\right]
\end{aligned}
$$




\section{Proof of Proposition 1}

We split the option payoff in two components:

1. The "contingent exercise payment", a claim with payoff

$$
C_{1}(Z, T, T . K)=-K I_{\left\{Z_{T} \geq K\right\}}=\left\{\begin{array}{ccc}
-K & \text { if } & Z_{T} \geq K \\
0 & \text { if } & Z_{T}<K
\end{array}\right.
$$

2. The "contingent receipt of the stock", a claim with payoff

$$
C_{2}(Z, T, T, K)=Z_{T} I_{\left\{Z_{T} \geq K\right\}}=\left\{\begin{array}{ccc}
Z_{T} & \text { if } \quad Z_{T} \geq K \\
0 & \text { if } \quad Z_{T}<K
\end{array}\right.
$$

We will compute the two components of the option:

$$
\begin{aligned}
& C_{1}(Z, 0, T, K)=E\left[e^{-r T} C_{1}(Z, T, T, K) \mid F_{t}\right]=-K e^{-r T} P\left(Z_{T} \geq K \backslash 49\right) \\
& C_{2}(Z, 0, T, K)=E\left[e^{-r T} C_{2}(Z, T, T, K) \mid F_{t}\right]=e^{-r T} E\left[Z_{T} \mid Z_{T} \geq K\right]
\end{aligned}
$$

Equation (8) implies

$$
\frac{\ln \left(e^{-\alpha_{Z} T} E\left(Z_{T}\right) / Z_{T}\right)+\left(\alpha_{Z}-\frac{1}{2} \sigma_{Z}^{2}\right) T}{\sigma_{Z} \sqrt{T}} \sim N(0,1)
$$

and, then, it is verified that

$$
P\left(Z_{T} \geq K\right)=N\left(d_{2}\right)
$$

where

$$
d_{2}=\frac{\ln \left(e^{-\alpha_{Z} T} E\left(Z_{T}\right) / K\right)+\left(\alpha_{Z}-\frac{1}{2} \sigma_{Z}^{2}\right) T}{\sigma_{Z} \sqrt{T}}
$$


Part 2 in Lemma 1 and a little algebra leads to

$$
E\left[Z_{T} \mid Z_{T} \geq K\right]=E\left(Z_{T}\right) N\left(d_{1}\right)
$$

where $d_{1}=d_{2}+\sigma_{Z} \sqrt{T}$.

Including (51)-(52) into (49)-(50), we obtain the final expression for the option price. 


\section{Proof of Lemma 4}

For $n \geq 2$, we have

$$
\begin{aligned}
V\left(\sum_{i=1}^{n} W_{t_{i}}\right) & =V\left(\sum_{i=1}^{n-1} W_{t_{i}}+W_{t_{n}}\right) \\
& =V\left(\sum_{i=1}^{n-1} W_{t_{i}}\right)+t_{n}+2 \sum_{i=1}^{n-1} \operatorname{Cov}\left(W_{t_{i}}, W_{t_{n}}\right) \\
& =V\left(\sum_{i=1}^{n-1} W_{t_{i}}\right)+n \Delta t+2 \sum_{i=1}^{n-1} i \Delta t \\
& =V\left(\sum_{i=1}^{n-1} W_{t_{i}}\right)+n^{2} \Delta t
\end{aligned}
$$

By induction, we get

$$
\begin{aligned}
& V\left(\sum_{i=1}^{n} W_{t_{i}}\right)=\sum_{i=1}^{n} i^{2} \Delta t=\frac{(n+1)\left(n+\frac{1}{2}\right) n}{3} \Delta t \\
& V\left(n W_{t_{n}}-\sum_{i=1}^{n} W_{t_{i}}\right) \\
& =V\left(n W_{t_{n}}\right)+V\left(\sum_{i=1}^{n} W_{t_{i}}\right)-2 \operatorname{Cov}\left(n W_{t_{n}}, \sum_{i=1}^{n} W_{t_{i}}\right) \\
& =n^{2} V\left(W_{t_{n}}\right)+\frac{(n+1)\left(n+\frac{1}{2}\right) n}{3} \Delta t-2 n \sum_{i=1}^{n} \operatorname{Cov}\left(W_{t_{n}}, W_{t_{i}}\right) \\
& =n^{2} t_{n}+\frac{(n+1)\left(n+\frac{1}{2}\right) n}{3} \Delta t-2 n \sum_{i=1}^{n} t_{i} \\
& =\left[n^{2}+\frac{(n+1)\left(n+\frac{1}{2}\right)}{3}-2 \sum_{i=1}^{n} i\right] n \Delta t \\
& =\frac{(n-1)\left(n-\frac{1}{2}\right) n}{3} \Delta t
\end{aligned}
$$




\section{Proof of Proposition 2}

These formulae are consequence of Proposition 1 and the moments of $Z_{n}$ that we get now:

- Departing from (13) and applying (2)-(3) in Lemma 1 and Lemma 4, we obtain the moments of $G_{n}$.

- The same procedure applied to (14) provides the moments of $S_{n} / G_{n}$.

- Comparing (14) and (15) and applying (4)-(5) in Lemma 1, we obtain the moments of $G_{n} / S_{n}$. 


\section{Proof of Lemma 5}

$$
\begin{aligned}
& V\left(\int_{0}^{T} W_{t} d t\right) \\
= & E\left(\left(\int_{0}^{T} W_{t} d t\right)^{2}\right)-\left(E\left(\int_{0}^{T} W_{t} d t\right)\right)^{2} \\
= & E\left(\left(\int_{0}^{T} W_{t} d t\right)^{2}\right)=E\left(\int_{0}^{T} W_{t} d t \int_{0}^{T} W_{s} d s\right) \\
= & E\left(\int_{0}^{T} W_{t}\left(\int_{0}^{T} W_{s} d s\right) d t\right)=E\left(\int_{0}^{T} \int_{0}^{T} W_{t} W_{s} d s d t\right) \\
= & \int_{0}^{T} \int_{0}^{T} E\left(W_{t} W_{s}\right) d s d t=\int_{0}^{T} \int_{0}^{T} \operatorname{Cov}\left(W_{t} W_{s}\right) d s d t \\
= & \int_{0}^{T} \int_{0}^{t} \operatorname{Cov}\left(W_{t} W_{s}\right) d s d t+\int_{0}^{T} \int_{t}^{T} \operatorname{Cov}\left(W_{t} W_{s}\right) d s d t \\
= & \int_{0}^{T} \int_{0}^{t} s d s d t+\int_{0}^{T} \int_{t}^{T} t d s d t \\
= & \frac{T^{3}}{3} \\
= & \frac{T^{3}}{3}-2 T \int_{0}^{T} t d t \\
= & V\left(T W_{T}\right)+V\left(\int_{0}^{T} W_{t} d t\right)-2 \operatorname{Cov}\left(T W_{T}, \int_{0}^{T} W_{t} d t\right) \\
& V\left(W_{T}-\int_{0}^{T} W_{t} d t\right) \\
= & \operatorname{Cov}\left(W_{T}, W_{t}\right) d t \\
= & \left.T^{3}{ }^{3}\right) \\
= & \\
= & \\
= &
\end{aligned}
$$




\section{Proof of Proposition 3}

These formulae are consequence of Proposition 1 and the moments of $Z_{T}$ that we obtain now:

- Departing from (16) and applying (2)-(3) in Lemma 1 and Lemma 5, we obtain the moments of $G_{T}$.

- The same procedure applied to (17) provides the moments of $S_{T} / G_{T}$.

- Comparing (17) and (18) and applying (4)-(5) in Lemma 1, we obtain the moments of $G_{T} / S_{T}$. 


\section{Proof of Lemma 6}

Using (12) and a little algebra, we have, for $a, b, k \in \mathbb{R}$,

$$
\begin{aligned}
& E\left(S_{i}^{a} S_{j}^{b} S_{n}^{k}\right) \\
= & S_{0}^{a+b+k} E\left(\exp \left\{\left(r-q-\frac{1}{2} \sigma^{2}\right)\left(a t_{i}+b t_{j}+k t_{n}\right)+\sigma\left(a W_{t_{i}}+b W_{t_{j}}+k W_{t_{n}}\right)\right\}\right) \\
= & S_{0}^{a} \exp \left\{\left(r-q+\frac{a-1}{2} \sigma^{2}\right) a i \Delta t\right\} S_{0}^{b} \exp \left\{\left(r-q+\frac{b-1}{2} \sigma^{2}\right) b j \Delta t\right\} \\
& \times S_{0}^{k} \exp \left\{\left(r-q+\frac{k-1}{2} \sigma^{2}\right) k n \Delta t\right\} \exp \left\{a b \sigma^{2} \min \{i, j\} \Delta t\right\} \\
& \times \exp \left\{k \sigma^{2}(a i+b j) \Delta t\right\}
\end{aligned}
$$

Several particular cases are the following:

$$
\begin{aligned}
& b=k=0 \Rightarrow E\left(S_{i}^{a}\right)=S_{0}^{a} \exp \left\{\left(r-q+\frac{a-1}{2} \sigma^{2}\right) a i \Delta t\right\} \\
& a=k=0 \Rightarrow E\left(S_{j}^{b}\right)=S_{0}^{b} \exp \left\{\left(r-q+\frac{b-1}{2} \sigma^{2}\right) b j \Delta t\right\} \\
& a=b=0 \Rightarrow E\left(S_{n}^{k}\right)=S_{0}^{k} \exp \left\{\left(r-q+\frac{k-1}{2} \sigma^{2}\right) k n \Delta t\right\}
\end{aligned}
$$

Plugging these expressions into (53), we get

$E\left(S_{i}^{a} S_{j}^{b} S_{n}^{k}\right)=E\left(S_{i}^{a}\right) E\left(S_{j}^{b}\right) E\left(S_{n}^{k}\right) \exp \left\{a b \sigma^{2} \min \{i, j\} \Delta t\right\} \exp \left\{k \sigma^{2}(a i+b j) \Delta t\right\}$

For $k=0$, we get

$$
E\left(S_{i}^{a} S_{j}^{b}\right)=E\left(S_{i}^{a}\right) E\left(S_{j}^{b}\right) \exp \left\{a b \sigma^{2} \min \{i, j\} \Delta t\right\}
$$

and, then,

$$
E\left(S_{i}^{a} S_{j}^{b} S_{n}^{k}\right)=E\left(S_{i}^{a} S_{j}^{b}\right) E\left(S_{n}^{k}\right) \exp \left\{k \sigma^{2}(a i+b j) \Delta t\right\}
$$

Using (54) with $a=1, b=k$ and (55) with $a=b=1$, we obtain $E\left(S_{i} S_{j}^{k}\right)$ and $E\left(S_{i} S_{j} S_{n}^{k}\right)$, respectively. 
- Mean of $A_{n} S_{n}^{k}$

$$
E\left(A_{n} S_{n}^{k}\right)=E\left(\frac{1}{n} \sum_{i=1}^{n} S_{i} S_{n}^{k}\right)=\frac{1}{n} \sum_{i=1}^{n} E\left(S_{i} S_{n}^{k}\right)
$$

Using (29) with $j=n$, we have

$$
\begin{aligned}
E\left(A_{n} S_{n}^{k}\right) & =\frac{1}{n} \sum_{i=1}^{n} E\left(S_{i}\right) E\left(S_{n}^{k}\right) e^{k \sigma^{2} i \Delta t}=\frac{1}{n} E\left(S_{n}^{k}\right) \sum_{i=1}^{n} S_{0} e^{\left(r-q+k \sigma^{2}\right) i \Delta t} \\
& =\frac{S_{0}}{n} E\left(S_{n}^{k}\right) h_{1}\left(r^{*}\right)
\end{aligned}
$$

with $h_{1}($.$) as given by (33) and r^{*}=r-q+k \sigma^{2}$.

- Mean of $A_{n}^{2} S_{n}^{k}$

$$
\begin{aligned}
E\left(A_{n}^{2} S_{n}^{k}\right) & =E\left(\left(\frac{1}{n} \sum_{i=1}^{n} S_{i}\right)^{2} S_{n}^{k}\right) \\
& =\left(\frac{1}{n}\right)^{2} E\left(\sum_{i, j=1}^{n} S_{i} S_{j} S_{n}^{k}\right)=\left(\frac{1}{n}\right)^{2} \sum_{i, j=1}^{n} E\left(S_{i} S_{j} S_{n}^{k}\right) \\
& =\left(\frac{1}{n}\right)^{2} E\left(S_{n}^{k}\right) \sum_{i, j=1}^{n} E\left(S_{i} S_{j}\right) e^{k \sigma^{2}(i+j) \Delta t}
\end{aligned}
$$

where the last equation results from (30).

We define

$$
z_{n}^{*}=\sum_{i, j=1}^{n} E\left(S_{i} S_{j}\right) e^{k \sigma^{2}(i+j) \Delta t}
$$

Then, we have

$$
\begin{aligned}
z_{n}^{*} & =\sum_{i, j=1}^{n-1} E\left(S_{i} S_{j}\right) e^{k \sigma^{2}(i+j) \Delta t}+2 \sum_{i=1}^{n} E\left(S_{i} S_{n}\right) e^{k \sigma^{2}(i+n) \Delta t}-E\left(S_{n} S_{n}\right) e^{2 k \sigma^{2} n \Delta t} \\
& =z_{n-1}^{*}+2 \sum_{i=1}^{n} E\left(S_{i}\right) E\left(S_{n}\right) e^{\sigma^{2} i \Delta t} e^{k \sigma^{2}(i+n) \Delta t}-E\left(S_{n}^{2}\right) e^{2 k \sigma^{2} n \Delta t}
\end{aligned}
$$


After some algebra, we get the recurrence law

$$
z_{n}^{*}=z_{n-1}^{*}+S_{0}^{2}\left[2 f_{1}\left(r^{*}+\sigma^{2}\right)\left(e^{\left(2 r^{*}+\sigma^{2}\right) n \Delta t}-e^{r^{*} n \Delta t}\right)-e^{\left(2 r^{*}+\sigma^{2}\right) n \Delta t}\right]
$$

If we compute $z_{1}^{*}$ either by its definition or using (57) and compare both results, we obtain $z_{0}^{*}=0$.

Using (57) for different values of $n$, we obtain

$$
z_{n}^{*}=S_{0}^{2}\left[2 f_{1}\left(r^{*}+\sigma^{2}\right)\left(h_{1}\left(2 r^{*}+\sigma^{2}\right)-h_{1}\left(r^{*}\right)\right)-h_{1}\left(2 r^{*}+\sigma^{2}\right)\right]
$$

Plugging this expression into (56), we have $E\left(A_{n}^{2} S_{n}^{k}\right)=\left(\frac{S_{0}}{n}\right)^{2} E\left(S_{n}^{k}\right)\left[2 f_{1}\left(r^{*}+\sigma^{2}\right)\left(h_{1}\left(2 r^{*}+\sigma^{2}\right)-h_{1}\left(r^{*}\right)\right)-h_{1}\left(2 r^{*}+\sigma^{2}\right)\right]$ 


\section{Proof of Lemma 7}

1. Moments of the arithmetic average $A_{n}$

(a) Mean of $A_{n}$ :

Apply (31) with $k=0$.

(b) Covariance of $A_{n}$ with $S_{n}$ :

Apply (31) for $k=1$ and (36).

(c) Variance of $A_{n}$ :

Apply (32) for $k=0$ and (36).

2. Moments of the variable $S_{n} / A_{n}$ :

Apply part 2 in Lemma 2 with $X=S_{n}, Y=A_{n}$.

3. Moments of the variable $A_{n} / S_{n}$

(a) Mean of $A_{n} / S_{n}$ :

Apply (28) for $i=n, k=-1$ and (31) for $k=-1$.

(b) Variance of $A_{n} / S_{n}$ :

Apply (28) for $i=n, k=-2$, (32) for $k=-2$ and (39). 


\section{Proof of Remark 1}

1. Replace $r=q-\sigma^{2}$ into (36)-(37) to obtain $E\left(A_{n}\right)$ and $\operatorname{Cov}\left(A_{n}, S_{n}\right)$.

To compute $V\left(A_{n}\right)$, we will need the following relationships, satisfied by the functions $h_{1}($.$) and f_{1}($.$) (see (33)-(34)):$

$$
\begin{aligned}
h_{1}(-a) & =\exp \{-(n+1) a \Delta t\} h_{1}(a) \\
f_{1}(-a) & =-\exp \{-a \Delta t\} f_{1}(a) \\
1+h_{1}(a) & =f_{1}(-a)\left[1-e^{(n+1) a \Delta t}\right] \\
f_{1}(b) & =\exp \{(b-a) \Delta t\} \frac{e^{a \Delta t}-1}{e^{b \Delta t}-1} f_{1}(a)
\end{aligned}
$$

Looking at (38), we need to compute

$$
f_{1}\left(r-q+\sigma^{2}\right)\left[h_{1}\left(2(r-q)+\sigma^{2}\right)-h_{1}(r-q)\right]
$$

Defining $x=r-q+\sigma^{2}$ and using (61), this expression becomes

$$
f_{1}(x) f_{1}\left(x-\sigma^{2}\right)\left[e^{x \Delta t} \frac{e^{\left(x-\sigma^{2}\right) \Delta t}-1}{e^{\left(2 x-\sigma^{2}\right) \Delta t}-1}\left(e^{\left(2 x-\sigma^{2}\right) n \Delta t}-1\right)-\left(e^{\left(2 x-\sigma^{2}\right) \Delta t}-1\right)\right]
$$

Taking limits when $x \rightarrow 0$ and applying (59) and some algebra, we obtain

$$
f_{1}\left(\sigma^{2}\right)\left[h_{1}\left(-\sigma^{2}\right)-n e^{-(n+1) \sigma^{2} \Delta t}\right]
$$

Replacing this result into (38) and using (58) and (60), we obtain

$$
V\left(A_{n}\right)=\left(\frac{S_{0}}{n}\right)^{2} f_{1}\left(\sigma^{2}\right) e^{-(n+1) \sigma^{2} \Delta t}\left[h_{1}\left(\sigma^{2}\right)-2 n+h_{1}\left(-\sigma^{2}\right)\right]
$$

It can be seen that, as expected, this variance is positive since

$$
h_{1}\left(\sigma^{2}\right)-2 n+h_{1}\left(-\sigma^{2}\right)=2 \sum_{i=1}^{n}\left(\cosh \left(\sigma^{2} i \Delta t\right)-1\right)>0
$$


2. Replace $r=q+\sigma^{2}$ into (39) and use $h_{1}(0)=n$ to obtain $E\left(A_{n} / S_{n}\right)$.

To compute $V\left(A_{n} / S_{n}\right)$, looking at (40), we need to compute

$$
f_{1}\left(r-q-\sigma^{2}\right)\left[h_{1}\left(2(r-q)-3 \sigma^{2}\right)-h_{1}\left(r-q-2 \sigma^{2}\right)\right]
$$

Defining $x=r-q-\sigma^{2}$ and applying the same procedure as in part 2 of this remark, this expression becomes

$$
f_{1}\left(\sigma^{2}\right)\left[h_{1}\left(-\sigma^{2}\right)-n e^{-(n+1) \sigma^{2} \Delta t}\right]
$$

Replacing this result into (40) and using (58), we obtain

$$
V\left(\frac{A_{n}}{S_{n}}\right)=\left(\frac{1}{n}\right)^{2}\left[\left(2 f_{1}\left(\sigma^{2}\right)-1\right)\left(e^{-\sigma^{2} \Delta t} h_{1}\left(\sigma^{2}\right)-n\right)-n(n-1)\right]
$$




\section{Proof of Lemma 8}

- Mean of $A_{T} S_{T}^{k}$

$$
E\left(A_{T} S_{T}^{k}\right)=E\left(\frac{1}{T} \int_{0}^{T} S_{t} d t S_{T}^{k}\right)=\frac{1}{T} \int_{0}^{T} E\left(S_{t} S_{T}^{k}\right) d t
$$

Using (29) with $j=n$, we have

$$
\begin{aligned}
E\left(A_{T} S_{T}^{k}\right) & =\frac{1}{T} \int_{0}^{T} E\left(S_{t}\right) E\left(S_{T}^{k}\right) \exp \left\{k \sigma^{2} t\right\} d t \\
& =\frac{1}{T} E\left(S_{T}^{k}\right) \int_{0}^{T} S_{0} \exp \{(r-q) t\} \exp \left\{k \sigma^{2} t\right\} d t \\
& =\frac{S_{0}}{T} E\left(S_{T}^{k}\right) \Phi\left(r^{*}\right)
\end{aligned}
$$

with $\Phi($.$) as given by (43) and r^{*}=r-q+k \sigma^{2}$.

- Mean of $A_{T}^{2} S_{T}^{k}$

Use (24) and apply (29)-(30). 


\section{Proof of Lemma 9}

1. Moments of the arithmetic average $A_{T}$

(a) Mean of $A_{T}$ :

Apply (41) with $k=0$.

(b) Covariance of $A_{T}$ with $S_{T}$ :

Apply (41) for $k=1$ and (44).

(c) Variance of $A_{T}$ :

Apply (42) for $k=0$ and (44).

2. Moments of the variable $S_{T} / A_{T}$

Apply part 2 in Lemma 2 with $X=S_{T}, Y=A_{T}$.

3. Moments of the variable $A_{T} / S_{T}$

(a) Mean of $A_{T} / S_{T}$

Apply (28) for $i=n, k=-1$ and (41) for $k=-1$.

(b) Variance of $A_{T} / S_{T}$

Apply (28) for $i=n, k=-2$, (42) for $k=-2$ and (47). 


\section{Proof of Remark 2}

1. Replace $r=q-\sigma^{2}$ into (44)-(45) to obtain $E\left(A_{T}\right)$ and $\operatorname{Cov}\left(A_{T}, S_{T}\right)$.

To compute $V\left(A_{T}\right)$, looking at (46), we need to compute

$$
\lim _{r \rightarrow q-\sigma^{2}} \frac{\Phi\left(2(r-q)+\sigma^{2}\right)-\Phi(r-q)}{r-q+\sigma^{2}}
$$

that is easily computed applying the L'Hôpital's rule. Plugging this result into (46) and using the relationship $\Phi(-x)=e^{-x T} \Phi(x)$, we obtain

$$
V\left(A_{T}\right)=\left(\frac{S_{0}}{T}\right)^{2} \frac{e^{-\sigma^{2} T}}{\sigma^{2}}\left[\Phi\left(\sigma^{2}\right)-2 T+\Phi\left(-\sigma^{2}\right)\right]
$$

It can be seen that, as expected, this variance is positive since

$$
\Phi\left(\sigma^{2}\right)-2 T+\Phi\left(-\sigma^{2}\right)=\frac{2}{\sigma^{2}}\left[\sinh \left(\sigma^{2} T\right)-\sigma^{2} T\right]>0
$$

2. Replace $r=q+\sigma^{2}$ into $(47)$ and use $\Phi(0)=T$ to obtain $E\left(A_{T} / S_{T}\right)$.

To compute $V\left(A_{T} / S_{T}\right)$, looking at (48), we need to compute

$$
\lim _{r \rightarrow q+\sigma^{2}} \frac{\Phi\left(2(r-q)-3 \sigma^{2}\right)-\Phi\left(r-q-2 \sigma^{2}\right)}{r-q-\sigma^{2}}
$$

Applying the L'Hôpital's rule and plugging the result into (48), we obtain

$$
V\left(\frac{A_{T}}{S_{T}}\right)=\left(\frac{1}{T}\right)^{2}\left[2 \frac{\Phi\left(\sigma^{2}\right)-T}{\sigma^{2}}-T^{2}\right]
$$




\section{References}

[1] Alziari, B., J.P. Decamps and P.F. Koehl (1997). A P.D.E. Approach to Asian options: Analytical and Numerical Evidence. J ournal of Banking and Finance, 21, 5, 613-640.

[2] Armata, K. (2001). Closed Form Solutions for Pricing Asian Options, mimeo.

[3] Bouaziz, L., E. Briys and M. Crouhy (1994). The pricing of forward-starting asian options. J ournal of Banking and Finance, $18,5,823-839$.

[4] Carverhill, A.P. and L.J. Clewlow (1990). Flexible Convolution. Risk, 3, 4, 25-29.

[5] Chung, S.L., M. Shackleton, and R. Wojakowski (2001). Efficient Quadratic Approximation of Floating Strike Asian Option Values, mimeo.

[6] Corwin, J., P.P. Boyle and K. Tan (1996). Quasi Monte Carlo Methods in Numerical Finance. Management Science, 42, 926938.

[7] Curran, V. (1994). Valuing Asian Options and Portfolio Options by Conditioning on the Geometric Mean Price. Management Science, 40, 12, 1705-1711. 
[8] De Schepper, A., M. Teunen and M. Goovaerts (1994). An Analytical Inversion of a Laplace Transform Related to Annuities Certain. Insurance: M athematics and E conomics, 14, 33-37.

[9] Dewynne, J. and P. Wilmott (1995a). Asian Options as Linear Complementary Problems: Analysis and Finite Difference Solutions. Advances in Futures and Options Research, 8, 145-173.

[10] Dewynne, J. and P. Wilmott (1995b). A Note on Average Rate Options with Discrete Sampling. SIAM J ournal of A pplied Mathematics, 55, 1, 267-276.

[11] Dinenis, E., D. Flamouris and J. Hatgioannies (2001). Valuing Exotic Options with Jump Diffusion Models: The Case of Asian Options, mimeo.

[12] Dufresne, D. (2000). Laguerre Series for Asian and Other Options. Mathematical Finance, 10, 4, 407-428.

[13] Eydeland, A. and H. Geman (1995). Domino Effect: Inverting the Laplace Transform. Risk, 8, 4, 65-67.

[14] Fu, M.C., D.B. Madan and T. Wang (1999). Pricing Continuous Asian Options: A Comparison of Monte Carlo and Laplace Transform Inversion Methods. J ournal of Computational Finance, $2,49-74$.

[15] Geman, H. and M. Yor (1993). Bessel Prices, Asian Options and Perpetuities. Mathematical Finance, 3, 4, 349-375. 
[16] Handley, J.C. (2000). Variable Purchase Options. Review of Derivatives Research, 4, 219-230.

[17] Hansen, A.T. and P.L. Jorgensen (2000). Analytical Valuation of American-Style Asian Options. Management Science, 46, 8, $1116-1136$

[18] Haykov, J. (1993). A Better Control Variate for Pricing Standard Asian Options. J ournal of Financial Engineering, 2, 207216.

[19] He, H. and A. Takahashi (1995-96). A Variable Reduction Technique for Pricing Average-Rate-Options. J apanese J ournal of Financial Economics, 1, 3-23.

[20] Jacques, M. (1996). On the Hedging Portfolio of Asian Options. ASTIN Bulletin, 26, 165-183.

[21] Jarrow, R. and A. Rudd (1982). Approximate Option Valuation for Arbitrary Stochastic Processes. J ournal of Financial Economics, 10, 347-369.

[22] Johnson, N.L. and S. Kotz (1970). Continuous Univariate Distributions-1. Distributions in Statistics, John Wiley \& Sons, New York.

[23] Ju, N. (1997). Fourier Transformation, Martingale, and the Pricing of Average Rate Derivatives, Ph.D. Thesis, University of California-Berkeley. 
[24] Kemna, A.G.Z. and T.C.F. Vorst (1990). A Pricing Method for Options based on Average Asset Values. J ournal of B anking and Finance, 14, 1, 113-129.

[25] Levy, E. (1992). Pricing European Average Rate Currency Options. J ournal of I nternational M oney and F inance, 11, 474-491.

[26] Levy, E. and S. Turnbull (1992). Average Intelligence. Risk, 5, 2, 53-59.

[27] Majumdar, M. and R. Radner (1991). Linear Models of Economic Survival under Production Uncertainty. E conomic Theory, $1,13-30$.

[28] Merton, R. (1973). Theory of Rational Option Pricing. Bell J ournal of Economics and Management Science, 4, 141-183.

[29] Merton, R. (1975). An Asymptotic Theory of Growth under Uncertainty. Review of E conomic Studies, 375-393.

[30] Milevsky, M.A. and S.E. Posner (1998). Asian Options, the Sum of Lognormals, and the Reciprocal Gamma Distribution. J ournal of Financial and Quantitative Analysis, 33, 3, 409-422.

[31] Mood, A.M, F.A. Graybill and D.C. Boes (1974). Introduction to the Theory of Statistics, Mc-Graw Hill Series in Probability and Statistics, Third Edition, New York. 
[32] Nielsen, J.A. and K. Sandmann (1996). The Pricing of Asian Options under Stochastic Interest Rates. Applied Mathematical Finance, 3, 209-236.

[33] Nielsen, J.A. and K. Sandmann (1998). Asian Exchange Rate Options under Stochastic Interest Rates: Pricing as a Sum of Delayed Payment Options, mimeo.

[34] Nielsen, J.A. and K. Sandmann (1999). Pricing of Asian Exchange Rate Options under Stochastic Interest Rates as a Sum of Delayed Payment Options, Technical Report, Department of Mathematical Sciences, University of Aarhus

[35] Nielsen, J.A. and K. Sandmann (2001). Pricing Bounds on Asian Options, mimeo.

[36] Posner, S.E. and M.A. Milevsky (1998). Valuing Exotic Options by Approximating the spd with Higher Moments. J ournal of Financial Engineering, 7, 109-125.

[37] Ritchken, P., L. Sankarasubramaniyan and A.M. Vijh (1993). The Valuation of Path Dependent Contracts on the Average. Management Science, 39, 10, 1202-1213.

[38] Rogers, L.C.G. and Z. Shi (1995). The Value of an Asian Option. J ournal of A pplied Probability, 32, 1077-1088.

[39] Shirakawa, H. (1999). Evaluation of the Asian Option by the Dual Martingale Measure. A sian-Pacific Financial Markets, 6, 183-194. 
[40] Shreve, S.E. and J. Vec̃er (2000). Options on a Traded Account, Vacation Calls, Vacation Puts and Passport Options, Working paper, Carnegie Mellon University.

[41] Stuart, A. and J.K. Ord (1987). Kendall's Advanced Theory of Statistics, Vol. I, Distribution Theory, Charles Griffin \& Company Limited, Fifth Edition, London.

[42] Turnbull, S.M. and L. M. Wakeman (1991). A Quick Algorithm for Pricing European Average Options. J ournal of F inancial and Quantitative Analysis, 26, 3, 377 -389.

[43] Vázquez-Abad, F. and D. Dufresne (1998). Accelerated Simulation for Pricing Asian Options, invited paper, Winter Simulation Conference Proceedings, 1493-1500.

[44] Vorst, T.C.F. (1990). Analytic Boundaries and Approximations of the Prices and Hedge Ratios of Average Exchange Rate Options. Working Paper, Econometric Institute, Erasmus University, Rotterdam.

[45] Vorst, T.C.F. (1992). Prices and Hedge Ratios of Average Exchange Rate Options. International Review of Financial Analysis, 1, 3, 179-193.

[46] Vorst, T.C.F. (1996). Averaging Options. In Handbook of Exotic Options, ed. by I. Nielken, Honeywood, Il, Irwin, 175-199.

[47] Yor, M. (1992). On some Exponential Functionals of Brownian Motion. Advances in A pplied Probability, 24, 509-531. 
[48] Yor, M. (1993). From Planar Brownian Windings to Asian Options. Insurance, Mathematics and E conomics, 13, 23-34.

[49] Zvan, R., A. Forsyth and K.R. Vetzal (1998). Robust Numerical Methods for PDE models of Asian Options. J ournal of Computational Finance, 1, 2, 39-78. 


\section{Footnotes}

1. For several examples in the real life, see Bouaziz et al (1994) and Vorst (1996).

2. This is one of the main assumptions in the Black-Scholes model. See Armata (2001) or Dinenis et al (2001) for valuation of Asian options outside the Black-Scholes framework.

3. As will be indicated later, the distribution of the continuous-time average is known, allowing us to obtain exact analytical expressions for option prices.

4. It may be worth noting that if these variables were perfectly correlated, then the average would be lognormal. Alternatively, if these variables are i.i.d., applying the central limit theorem, the distribution of the average would converge to the normal one.

5. Other examples are Dewynne and Wilmott (1995a, 1995b), He and Takahashi (199596), Zvan et al (1998) or Shreve and Vec̃er (2000).

6. See also Haykov (1993), Corwin et al (1996) and Nielsen and Sandmann (1996).

7. See, for example, Levy (1992), Hansen and Jorgensen (2000) and Dinenis et al (2001).

8. Merton (1975) and Majumdar and Radner (1991) are examples of papers in the economic literature that deal with the gamma distribution.

9. For completeness, this table includes the geometric average.

10. Recall that these prices have been multiplied by 100 .

11. For completeness, this table includes the stock price and its geometric average. The formula for the geometric Asian option was first derived by Kemna and Vorst (1990).

12. It can be shown that for $\sigma$ large enough $\left(\sigma>\sqrt{6 / 5} \sqrt{2 T \ln \left(S_{0}\right)+3(r-q)}\right)$, this expected value is higher than $E\left(S_{T}\right)$.

13. Analogous notation is employed for the approximating distribution $A$.

14. We use 10,000 simulations and antithetic variables to reduce standard errors. 
Table 1. Geometric Call option prices.

\begin{tabular}{|c|c|c|c|c|c|c|c|c|}
\hline \multicolumn{3}{|c|}{ Parameters } & Asset & \multicolumn{5}{|c|}{ Number of monitoring dates $(n)$} \\
\hline$\sigma(\%)$ & $T$ & $K$ & $Z_{n}$ & 1 & 10 & 100 & 1,000 & $\infty$ \\
\hline \multirow[t]{4}{*}{20} & 0.5 & 0.8 & $S_{n}$ & 22.576 & 22.576 & 22.576 & 22.576 & 22.576 \\
\hline & & & $G_{n}$ & 22.576 & 20.696 & 20.561 & 20.548 & 20.546 \\
\hline & & & $S_{n} / G_{n}$ & 19.025 & 20.400 & 20.531 & 20.545 & 20.546 \\
\hline & & & $G_{n} / S_{n}$ & 19.025 & 18.133 & 18.161 & 18.166 & 18.166 \\
\hline \multirow[t]{4}{*}{20} & 1 & 0.8 & $S_{n}$ & 25.187 & 25.187 & 25.187 & 25.187 & 25.187 \\
\hline & & & $G_{n}$ & 25.187 & 21.361 & 21.084 & 21.056 & 21.053 \\
\hline & & & $S_{n} / G_{n}$ & 18.097 & 20.756 & 21.023 & 21.050 & 21.053 \\
\hline & & & $G_{n} / S_{n}$ & 18.097 & 16.491 & 16.580 & 16.593 & 16.594 \\
\hline \multirow[t]{4}{*}{40} & 0.5 & 0.8 & $S_{n}$ & 24.801 & 24.801 & 24.801 & 24.801 & 24.801 \\
\hline & & & $G_{n}$ & 24.801 & 20.766 & 20.558 & 20.538 & 20.536 \\
\hline & & & $S_{n} / G_{n}$ & 19.025 & 20.332 & 20.514 & 20.534 & 20.536 \\
\hline & & & $G_{n} / S_{n}$ & 19.025 & 20.266 & 20.908 & 20.979 & 20.987 \\
\hline \multirow[t]{4}{*}{20} & 0.5 & 1.1 & $S_{n}$ & 3.175 & 3.175 & 3.175 & 3.175 & 3.175 \\
\hline & & & $G_{n}$ & 3.175 & 0.905 & 0.737 & 0.721 & 0.719 \\
\hline & & & $S_{n} / G_{n}$ & 0 & 0.549 & 0.701 & 0.717 & 0.719 \\
\hline & & & $G_{n} / S_{n}$ & 0 & 0.282 & 0.374 & 0.384 & 0.385 \\
\hline
\end{tabular}

Prices are multiplied by 100 . The interest rate is $10 \%$ and the dividend yield $3 \%$. For options on $S_{n}$ and $G_{n}$ we take $S_{0}=1$. For options on $G_{n}$, derivative prices can be computed with the Merton's (1973) formula when $n=1$, and with the Kemna and Vorst's (1990) formula when $n=\infty$. 
Table 2. Geometric Put option prices.

\begin{tabular}{|ccc|l|ccccc|}
\hline \multicolumn{2}{|c|}{ Parameters } & Asset & \multicolumn{5}{|c|}{ Number of monitoring dates $(n)$} \\
\hline$\sigma(\%)$ & $T$ & $K$ & $Z_{n}$ & 1 & 10 & 100 & 1,000 & $\infty$ \\
\hline 20 & 0.5 & 1.0 & $S_{n}$ & 3.930 & 3.930 & 3.930 & 3.930 & 3.930 \\
\hline & & & $G_{n}$ & 3.930 & 2.591 & 2.439 & 2.423 & 2.422 \\
& & & $S_{n} / G_{n}$ & 0 & 2.250 & 2.405 & 2.420 & 2.422 \\
& & & $G_{n} / S_{n}$ & 0 & 3.321 & 3.516 & 3.535 & 3.537 \\
\hline 20 & 1 & 1.0 & $S_{n}$ & 4.639 & 4.639 & 4.639 & 4.639 & 4.639 \\
\hline & & & $G_{n}$ & 4.639 & 3.135 & 2.955 & 2.937 & 2.935 \\
& & & $S_{n} / G_{n}$ & 0 & 2.732 & 2.915 & 2.933 & 2.935 \\
& & & $G_{n} / S_{n}$ & 0 & 4.724 & 4.976 & 5.000 & 5.002 \\
\hline 40 & 0.5 & 1.0 & $S_{n}$ & 9.277 & 9.277 & 9.277 & 9.277 & 9.277 \\
\hline & & & $G_{n}$ & 9.277 & 6.149 & 5.763 & 5.724 & 5.719 \\
& & & $S_{n} / G_{n}$ & 0 & 5.283 & 5.676 & 5.715 & 5.719 \\
& & & $G_{n} / S_{n}$ & 0 & 5.314 & 5.493 & 5.508 & 5.510 \\
\hline 20 & 0.5 & 1.1 & $S_{n}$ & 9.300 & 9.300 & 9.300 & 9.300 & 9.300 \\
\hline & & & $G_{n}$ & 9.300 & 8.753 & 8.712 & 8.714 & 8.713 \\
& & & $S_{n} / G_{n}$ & 9.512 & 8.688 & 8.710 & 8.713 & 8.713 \\
& & & $G_{n} / S_{n}$ & 9.512 & 10.691 & 10.759 & 10.765 & 10.766 \\
\hline
\end{tabular}

Prices are multiplied by 100 . The interest rate is $10 \%$ and the dividend yield $3 \%$. For options on $S_{n}$ and $G_{n}$ we take $S_{0}=1$. For options on $G_{n}$, derivative prices can be computed with the Merton's (1973) formula when $n=1$, and with the Kemna and Vorst's (1990) formula when $n=\infty$. 
Table 3. Arithmetic Call option prices.

\begin{tabular}{|c|c|c|c|c|c|c|c|c|}
\hline \multicolumn{3}{|c|}{ Parameters } & \multirow{2}{*}{$\begin{array}{r}\text { Asset } \\
Z_{n}\end{array}$} & \multicolumn{5}{|c|}{ Number of monitoring dates $(n)$} \\
\hline$\sigma(\%)$ & $T$ & $K$ & & 1 & 10 & 100 & 1,000 & $\infty$ \\
\hline \multirow[t]{9}{*}{20} & 0.5 & 0.8 & $A_{n} \mathrm{MC}$ & 22.551 & 20.737 & 20.731 & 20.723 & - \\
\hline & & & $A_{n} \mathrm{~W}$ & 22.576 & 20.885 & 20.729 & 20.714 & 20.712 \\
\hline & & & $A_{n} \mathrm{GD}$ & 22.535 & 20.883 & 20.728 & 20.711 & 20.711 \\
\hline & & & $S_{n} / A_{n} \mathrm{MC}$ & 19.025 & 20.329 & 20.381 & 20.377 & - \\
\hline & & & $S_{n} / A_{n} \mathrm{~W}$ & 19.025 & 20.209 & 20.360 & 20.375 & 20.377 \\
\hline & & & $S_{n} / A_{n} \mathrm{GD}$ & 19.025 & 20.208 & 20.358 & 20.374 & 20.375 \\
\hline & & & $A_{n} / S_{n} \mathrm{MC}$ & 19.025 & 18.332 & 18.317 & 18.319 & - \\
\hline & & & $A_{n} / S_{n} \mathrm{~W}$ & 19.025 & 18.389 & 18.330 & 18.324 & 18.324 \\
\hline & & & $A_{n} / S_{n} \mathrm{GD}$ & 19.025 & 18.388 & 18.327 & 18.321 & 18.321 \\
\hline \multirow[t]{9}{*}{20} & 1 & 0.8 & $A_{n} \mathrm{MC}$ & 25.089 & 21.431 & 21.366 & 21.387 & - \\
\hline & & & $A_{n} \mathrm{~W}$ & 25.187 & 21.736 & 21.418 & 21.387 & 21.384 \\
\hline & & & $A_{n} \mathrm{GD}$ & 25.059 & 21.716 & 21.404 & 21.373 & 21.370 \\
\hline & & & $S_{n} / A_{n} \mathrm{MC}$ & 18.097 & 20.642 & 20.730 & 20.766 & - \\
\hline & & & $S_{n} / A_{n} \mathrm{~W}$ & 18.097 & 20.376 & 20.682 & 20.713 & 20.717 \\
\hline & & & $S_{n} / A_{n} \mathrm{GD}$ & 18.097 & 20.366 & 20.667 & 20.698 & 20.701 \\
\hline & & & $A_{n} / S_{n} \mathrm{MC}$ & 18.097 & 16.841 & 16.884 & 16.899 & - \\
\hline & & & $A_{n} / S_{n} \mathrm{~W}$ & 18.097 & 16.964 & 16.887 & 16.880 & 16.883 \\
\hline & & & $A_{n} / S_{n} \mathrm{GD}$ & 18.097 & 16.947 & 16.862 & 16.855 & 16.857 \\
\hline
\end{tabular}

Prices are multiplied by 100 . The interest rate is $10 \%$ and the dividend yield $3 \%$. For options on $A_{n}$ we take $S_{0}=1$. MC, W and GD refer to Monte Carlo simulation, Wilkinson approximation and gamma distribution, respectively. 
Table 3. Arithmetic Call option prices (cont.).

\begin{tabular}{|ccc|l|ccccc|}
\hline \multicolumn{3}{|c|}{ Parameters } & \multicolumn{1}{|c|}{ Asset } & \multicolumn{5}{|c|}{ Number of monitoring dates $(n)$} \\
\hline$\sigma(\%)$ & $T$ & $K$ & $Z_{n}$ & 1 & 10 & 100 & 1,000 & $\infty$ \\
\hline 40 & 0.5 & 0.8 & $A_{n} \mathrm{MC}$ & 24.687 & 21.141 & 21.111 & 21.206 & - \\
& & & $A_{n} \mathrm{~W}$ & 24.801 & 21.468 & 21.188 & 21.161 & 21.158 \\
& & & $A_{n} \mathrm{GD}$ & 24.400 & 21.358 & 21.099 & 21.074 & 21.071 \\
\hline & & $S_{n} / A_{n} \mathrm{MC}$ & 19.025 & 19.853 & 20.011 & 19.987 & - \\
& & $S_{n} / A_{n} \mathrm{~W}$ & 19.025 & 19.641 & 19.918 & 19.947 & 19.951 \\
& & $S_{n} / A_{n} \mathrm{GD}$ & 19.025 & 19.562 & 19.819 & 19.846 & 19.849 \\
\hline & & $A_{n} / S_{n} \mathrm{MC}$ & 19.025 & 21.432 & 21.620 & 21.535 & - \\
& & $A_{n} / S_{n} \mathrm{~W}$ & 19.025 & 21.276 & 21.569 & 21.599 & 21.620 \\
& & $A_{n} / S_{n} \mathrm{GD}$ & 19.025 & 21.213 & 21.489 & 21.517 & 21.535 \\
\hline \multirow{4}{*}{0.5} & 1.1 & $A_{n} \mathrm{MC}$ & 3.141 & 0.802 & 0.756 & 0.757 & - \\
& & $A_{n} \mathrm{~W}$ & 3.176 & 0.933 & 0.778 & 0.761 & 0.759 \\
& & $A_{n} \mathrm{GD}$ & 3.198 & 0.980 & 0.802 & 0.785 & 0.782 \\
\hline & & $S_{n} / A_{n} \mathrm{MC}$ & 0 & 0.593 & 0.612 & 0.682 & - \\
& $S_{n} / A_{n} \mathrm{~W}$ & 0 & 0.528 & 0.681 & 0.698 & 0.699 \\
& $S_{n} / A_{n} \mathrm{GD}$ & 0 & 0.549 & 0.705 & 0.721 & 0.722 \\
\hline & $A_{n} / S_{n} \mathrm{MC}$ & 0 & 0.389 & 0.404 & 0.413 & - \\
& $A_{n} / S_{n} \mathrm{~W}$ & 0 & 0.302 & 0.387 & 0.396 & 0.401 \\
& $A_{n} / S_{n} \mathrm{GD}$ & 0 & 0.320 & 0.409 & 0.419 & 0.424 \\
\hline
\end{tabular}

Prices are multiplied by 100 . The interest rate is $10 \%$ and the dividend yield $3 \%$. For options on $A_{n}$ we take $S_{0}=1$. MC, W and GD refer to Monte Carlo simulation, Wilkinson approximation and gamma distribution, respectively. 
Table 4. Arithmetic Put option prices.

\begin{tabular}{|c|c|c|c|c|c|c|c|c|}
\hline \multicolumn{3}{|c|}{ Parameters } & \multirow{2}{*}{$\begin{array}{l}\text { Asset } \\
Z_{n}\end{array}$} & \multicolumn{5}{|c|}{ Number of monitoring dates $(n)$} \\
\hline$\sigma(\%)$ & $T$ & $K$ & & 1 & 10 & 100 & 1,000 & $\infty$ \\
\hline \multirow[t]{9}{*}{20} & 0.5 & 1.0 & $A_{n} \mathrm{MC}$ & 3.930 & 2.331 & 2.417 & 2.387 & - \\
\hline & & & $A_{n} \mathrm{~W}$ & 3.930 & 2.530 & 2.387 & 2.372 & 2.371 \\
\hline & & & $A_{n} \mathrm{GD}$ & 3.868 & 2.511 & 2.371 & 2.357 & 2.356 \\
\hline & & & $S_{n} / A_{n} \mathrm{MC}$ & 0 & 2.365 & 2.477 & 2.512 & - \\
\hline & & & $S_{n} / A_{n} \mathrm{~W}$ & 0 & 2.342 & 2.490 & 2.505 & 2.506 \\
\hline & & & $S_{n} / A_{n} \mathrm{GD}$ & 0 & 2.330 & 2.475 & 2.491 & 2.494 \\
\hline & & & $A_{n} / S_{n} \mathrm{MC}$ & 0 & 3.328 & 3.440 & 3.408 & - \\
\hline & & & $A_{n} / S_{n} \mathrm{~W}$ & 0 & 3.176 & 3.417 & 3.440 & 3.451 \\
\hline & & & $A_{n} / S_{n}$ GD & 0 & 3.175 & 3.415 & 3.440 & 3.449 \\
\hline \multirow[t]{9}{*}{20} & 1 & 1.0 & $A_{n} \mathrm{MC}$ & 4.639 & 2.821 & 2.771 & 2.872 & - \\
\hline & & & $A_{n} \mathrm{~W}$ & 4.639 & 3.038 & 2.875 & 2.859 & 2.857 \\
\hline & & & $A_{n} \mathrm{GD}$ & 4.479 & 2.991 & 2.834 & 2.819 & 2.817 \\
\hline & & & $S_{n} / A_{n} \mathrm{MC}$ & 0 & 3.043 & 2.994 & 3.055 & - \\
\hline & & & $S_{n} / A_{n} \mathrm{~W}$ & 0 & 2.913 & 3.086 & 3.103 & 3.105 \\
\hline & & & $S_{n} / A_{n} \mathrm{GD}$ & 0 & 2.884 & 3.051 & 3.068 & 3.069 \\
\hline & & & $A_{n} / S_{n} \mathrm{MC}$ & 0 & 4.736 & 4.854 & 4.817 & - \\
\hline & & & $A_{n} / S_{n} \mathrm{~W}$ & 0 & 4.436 & 4.775 & 4.809 & 4.833 \\
\hline & & & $A_{n} / S_{n}$ GD & 0 & 4.433 & 4.770 & 4.803 & 4.827 \\
\hline
\end{tabular}

Prices are multiplied by 100 . The interest rate is $10 \%$ and the dividend yield $3 \%$. For options on $A_{n}$ we take $S_{0}=1$. MC, W and GD refer to Monte Carlo simulation, Wilkinson approximation and gamma distribution, respectively. 
Table 4. Arithmetic Put option prices (cont.).

\begin{tabular}{|c|c|c|c|c|c|c|c|c|}
\hline \multicolumn{3}{|c|}{ Parameters } & Asset & \multicolumn{5}{|c|}{ Number of monitoring dates $(n)$} \\
\hline$\sigma(\%)$ & $T$ & $K$ & $Z_{n}$ & 1 & 10 & 100 & 1,000 & $\infty$ \\
\hline \multirow[t]{9}{*}{40} & 0.5 & 1.0 & $A_{n} \mathrm{MC}$ & 9.277 & 5.465 & 5.402 & 5.554 & - \\
\hline & & & $A_{n} \mathrm{~W}$ & 9.277 & 5.874 & 5.525 & 5.490 & 5.487 \\
\hline & & & $A_{n} \mathrm{GD}$ & 8.971 & 5.792 & 5.457 & 5.423 & 5.419 \\
\hline & & & $S_{n} / A_{n} \mathrm{MC}$ & 0 & 5.855 & 6.000 & 5.988 & - \\
\hline & & & $S_{n} / A_{n} \mathrm{~W}$ & 0 & 5.837 & 6.202 & 6.238 & 6.242 \\
\hline & & & $S_{n} / A_{n} \mathrm{GD}$ & 0 & 5.794 & 6.147 & 6.182 & 6.186 \\
\hline & & & $A_{n} / S_{n} \mathrm{MC}$ & 0 & 5.078 & 5.202 & 5.201 & - \\
\hline & & & $A_{n} / S_{n} \mathrm{~W}$ & 0 & 4.880 & 5.193 & 5.224 & 5.290 \\
\hline & & & $A_{n} / S_{n} \mathrm{GD}$ & 0 & 4.822 & 5.124 & 5.154 & 5.217 \\
\hline \multirow[t]{9}{*}{20} & 0.5 & 1.1 & $A_{n} \mathrm{MC}$ & 9.300 & 8.571 & 8.581 & 8.576 & - \\
\hline & & & $A_{n} \mathrm{~W}$ & 9.300 & 8.613 & 8.589 & 8.588 & 8.588 \\
\hline & & & $A_{n} \mathrm{GD}$ & 9.322 & 8.639 & 8.613 & 8.611 & 8.611 \\
\hline & & & $S_{n} / A_{n} \mathrm{MC}$ & 9.512 & 8.838 & 8.827 & 8.829 & - \\
\hline & & & $S_{n} / A_{n} \mathrm{~W}$ & 9.512 & 8.858 & 8.862 & 8.863 & 8.863 \\
\hline & & & $S_{n} / A_{n} \mathrm{GD}$ & 9.512 & 8.879 & 8.886 & 8.887 & 8.887 \\
\hline & & & $A_{n} / S_{n} \mathrm{MC}$ & 9.512 & 10.586 & 10.626 & 10.617 & - \\
\hline & & & $A_{n} / S_{n} \mathrm{~W}$ & 9.512 & 10.453 & 10.603 & 10.618 & 10.623 \\
\hline & & & $A_{n} / S_{n} \mathrm{GD}$ & 9.512 & 10.472 & 10.625 & 10.640 & 10.646 \\
\hline
\end{tabular}

Prices are multiplied by 100 . The interest rate is $10 \%$ and the dividend yield $3 \%$. For options on $A_{n}$ we take $S_{0}=1$. MC, W and GD refer to Monte Carlo simulation, Wilkinson approximation and gamma distribution, respectively. 


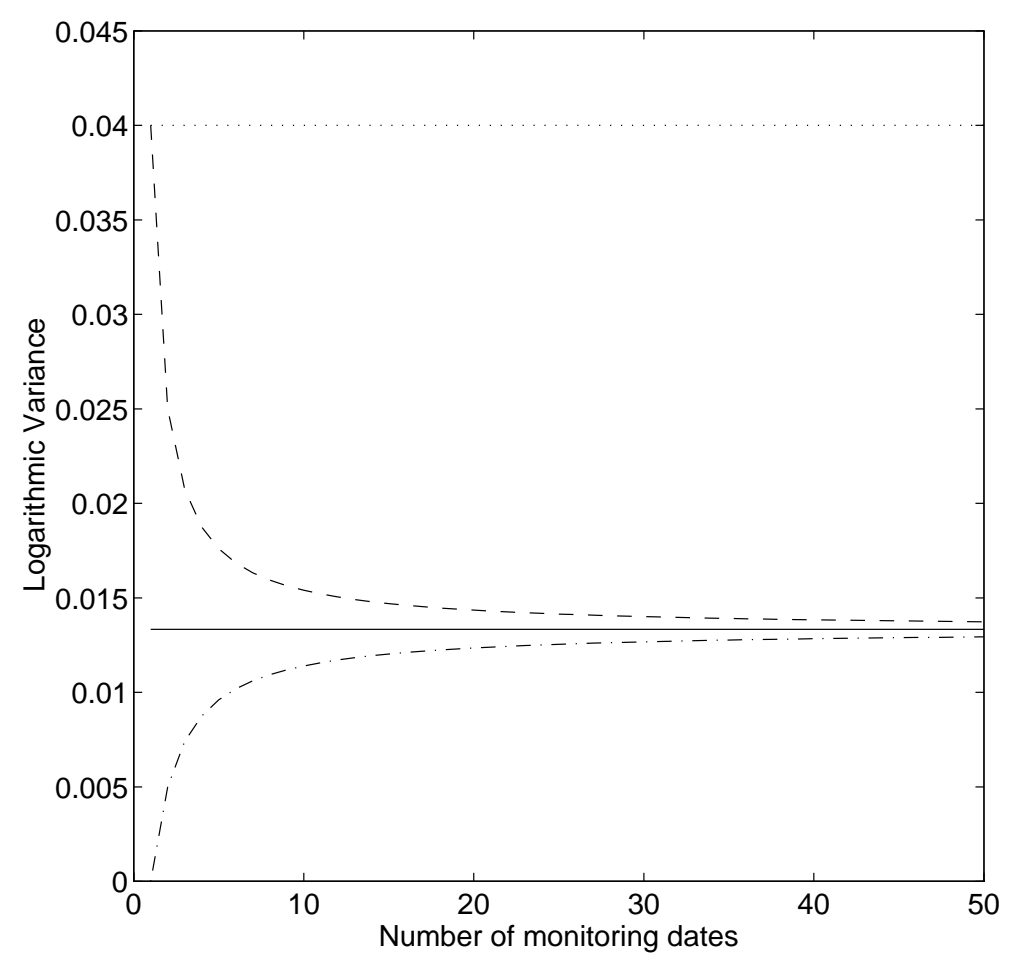

Figure 1: Plot of the logarithmic variance $\left(\sigma_{Z}^{2} T\right)$ as function of $n$. The parameter values are $\sigma=0.2$ and $T=1$. The figure depicts the logarithmic variance of the stock price (dotted line), $G_{n}$ (dashed line), $G_{T}$ (solid line), $S_{n} / G_{n}$ (dotted-dashed line), and $G_{n} / S_{n}$. These values are given by the following table:

\begin{tabular}{c|c}
$Z_{n}$ & $\sigma_{Z}^{2} T$ \\
\hline$S_{n}$ & $\sigma^{2} T$ \\
$G_{n}$ & $\frac{(n+1)\left(n+\frac{1}{2}\right)}{3 n^{2}} \sigma^{2} T$ \\
$S_{n} / G_{n}$ & $\frac{(n-1)\left(n-\frac{1}{2}\right)}{3 n^{2}} \sigma^{2} T$ \\
$G_{n} / S_{n}$ & $\frac{(n-1)\left(n-\frac{1}{2}\right)}{3 n^{2}} \sigma^{2} T$
\end{tabular}




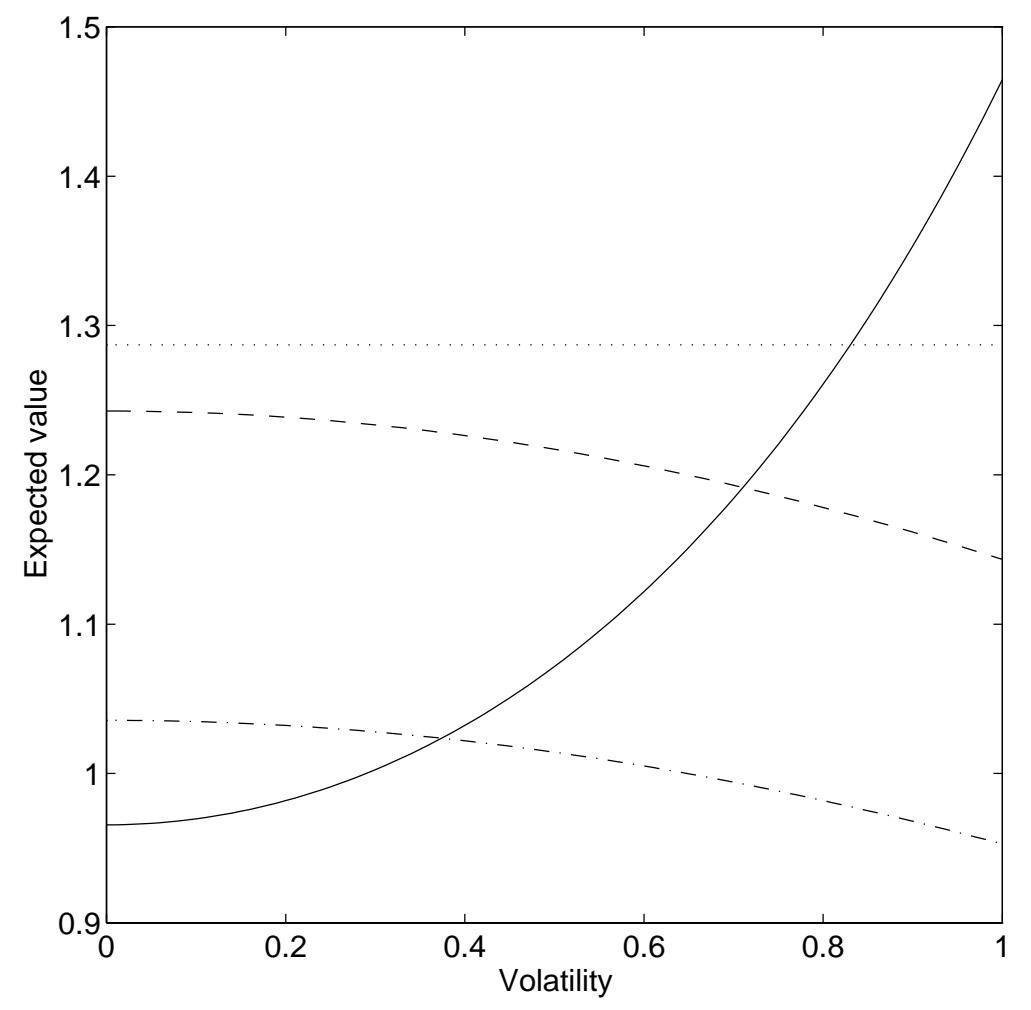

Figure 2: Plot of the expected value $E\left(Z_{T}\right)$ as function of $\sigma^{2}$. The parameter values are $r=10 \%, q=3 \%, \sigma=0.2$ and $T=1$. We assume $S_{0}=1.2$. The figure depicts the expected values of the stock price (dotted line), $G_{T}$ (dashed line), $S_{T} / G_{T}$ (dotted-dashed line), and $G_{T} / S_{T}$ (solid line). These values are given by the following table:

\begin{tabular}{c|c}
$Z_{T}$ & $E\left(Z_{T}\right)$ \\
\hline$S_{T}$ & $S_{0} \exp \{(r-q) T\}$ \\
$G_{T}$ & $S_{0} \exp \left\{\frac{1}{2}\left(r-q-\frac{1}{6} \sigma^{2}\right) T\right\}$ \\
$S_{T} / G_{T}$ & $\exp \left\{\frac{1}{2}\left(r-q-\frac{1}{6} \sigma^{2}\right) T\right\}$ \\
$G_{T} / S_{T}$ & $\exp \left\{-\frac{1}{2}\left(r-q-\frac{5}{6} \sigma^{2}\right) T\right\}$
\end{tabular}



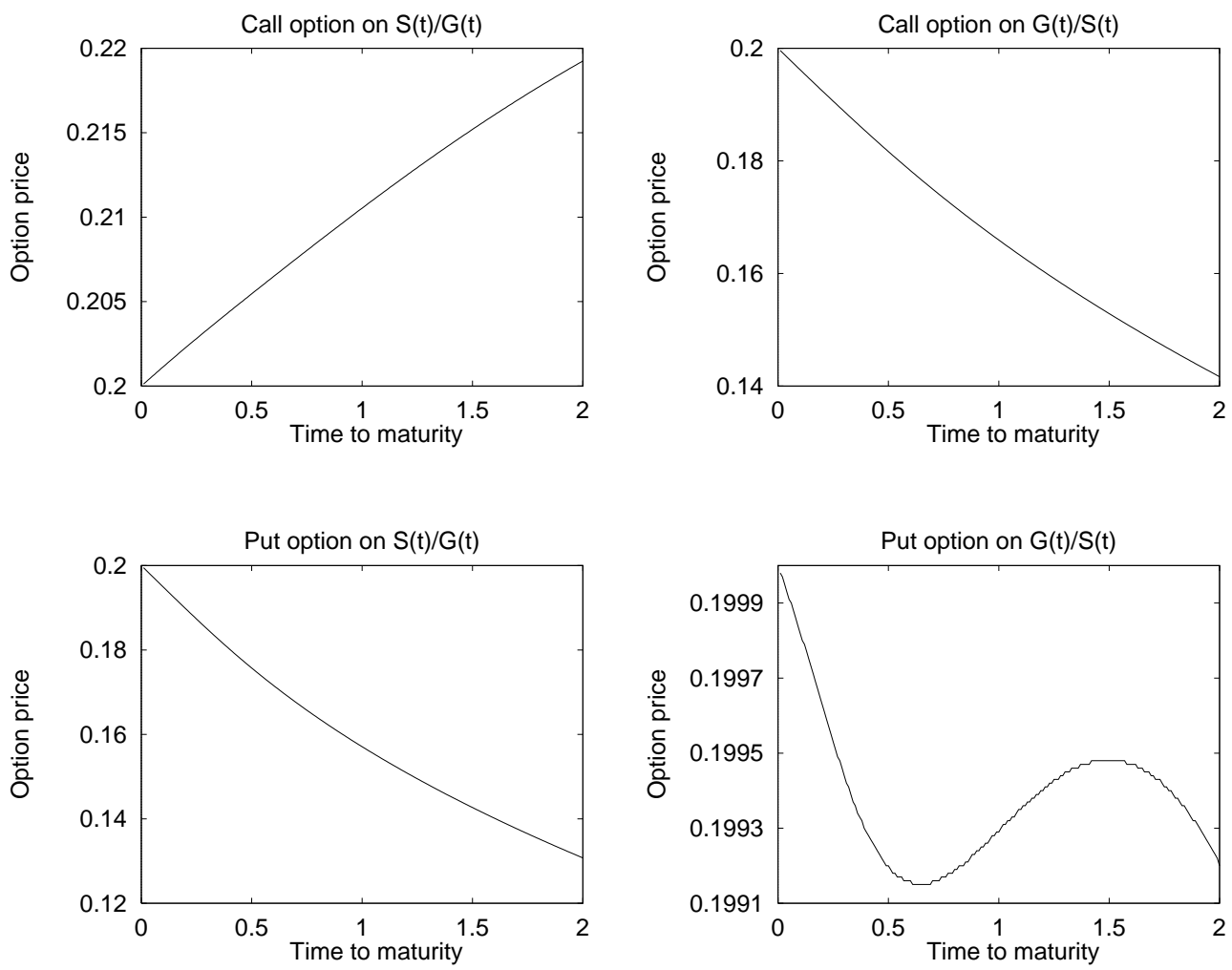

Figure 3: Geometric Australian option prices as a function of time to maturity. The exercise price is $K=0.8$ for calls and $K=1.2$ for puts. The other parameter values are: $r=0.1, q=0.03, \sigma=0.2$, and $n=\infty$. 

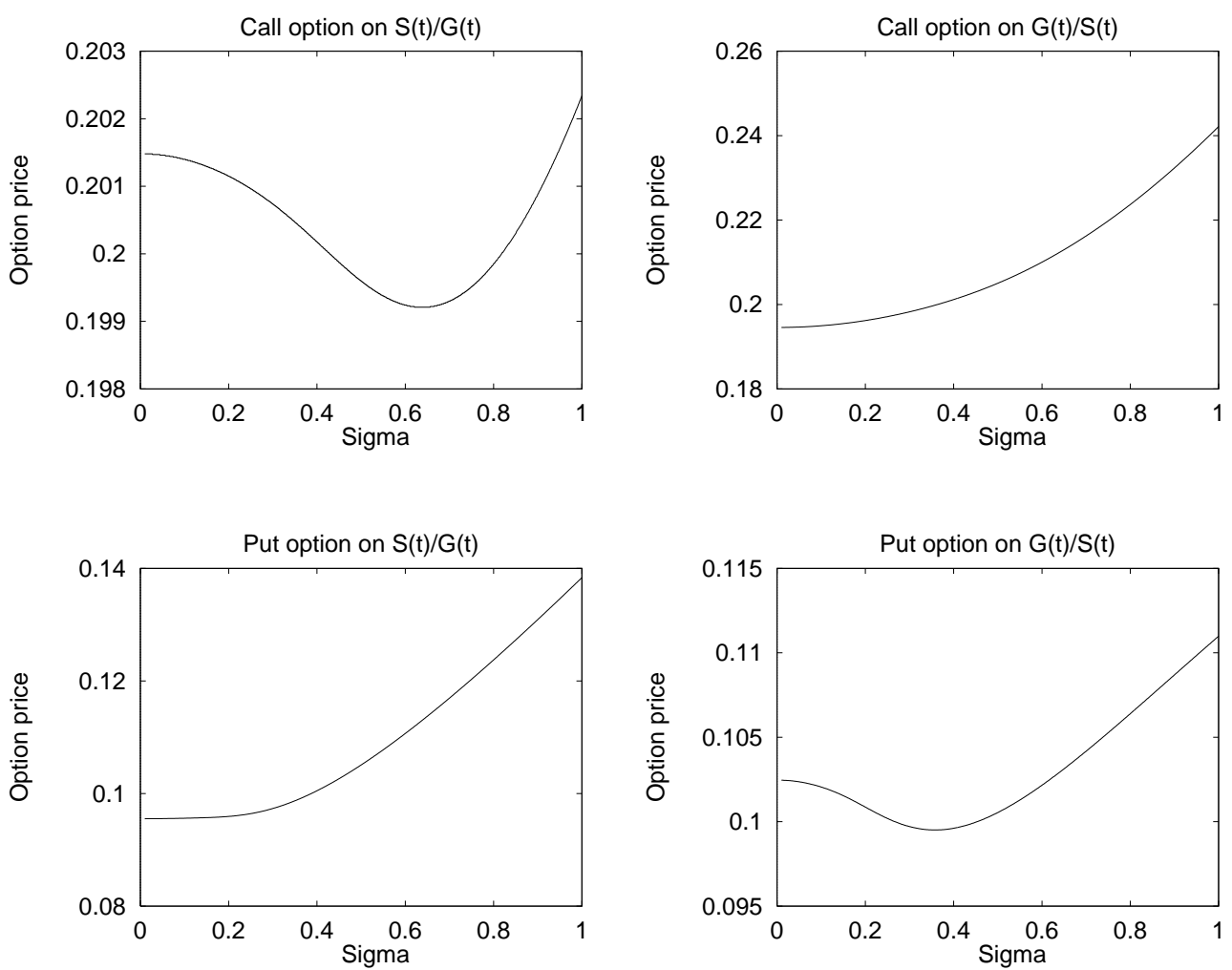

Figure 4: Geometric Australian option prices as a function of volatility $(\sigma)$. The exercise price is $K=0.8$ for calls and $K=1.1$ for puts. The other parameter values are: $r=0.1, q=0.03, T=0.1$, and $n=\infty$. 


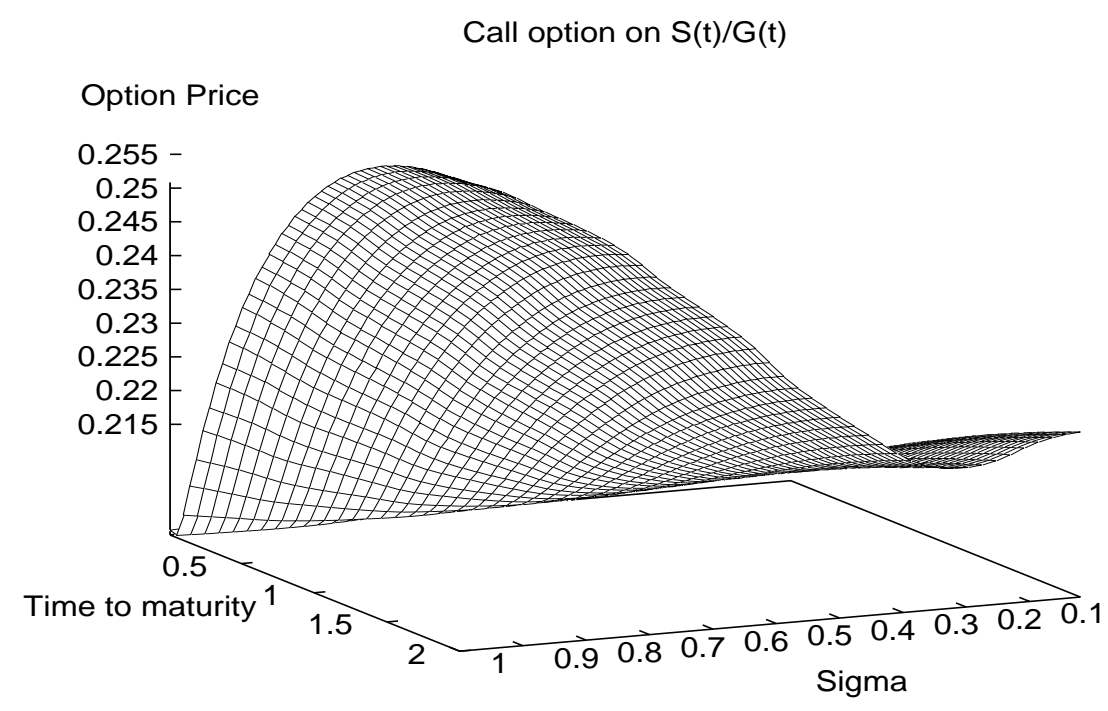

Put option on $\mathrm{G}(\mathrm{t}) / \mathrm{S}(\mathrm{t})$

Option Price

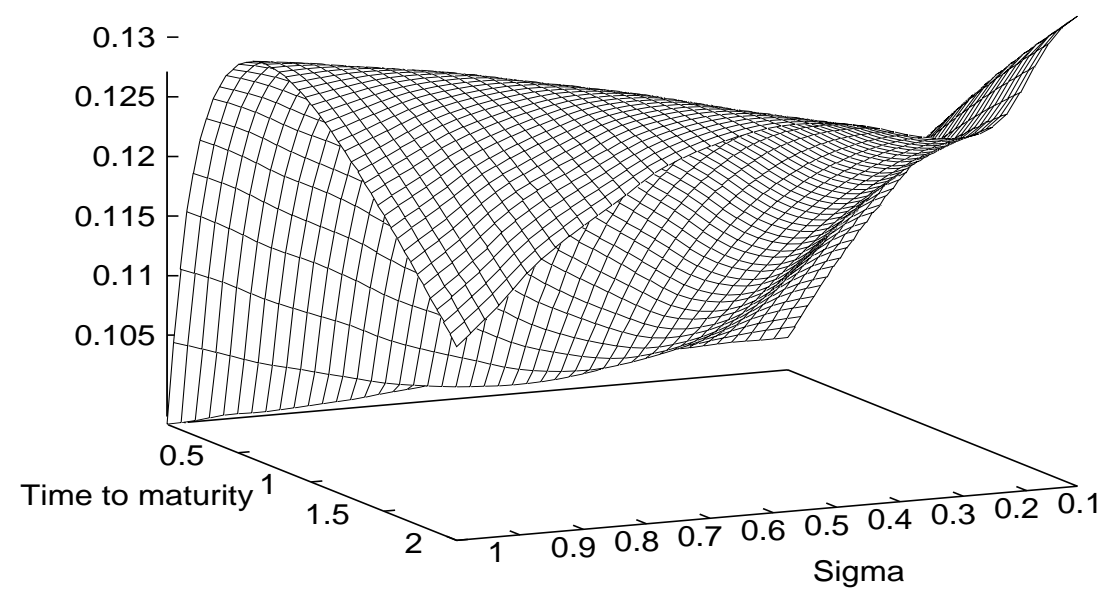

Figure 5: Geometric Australian option prices as a function of time to maturity and volatility $(\sigma)$. The exercise price is $K=0.8$ for the call option and $K=1.1$ for the put option. The other parameter values are: $r=0.1, q=0.03$, and $n=\infty$. 


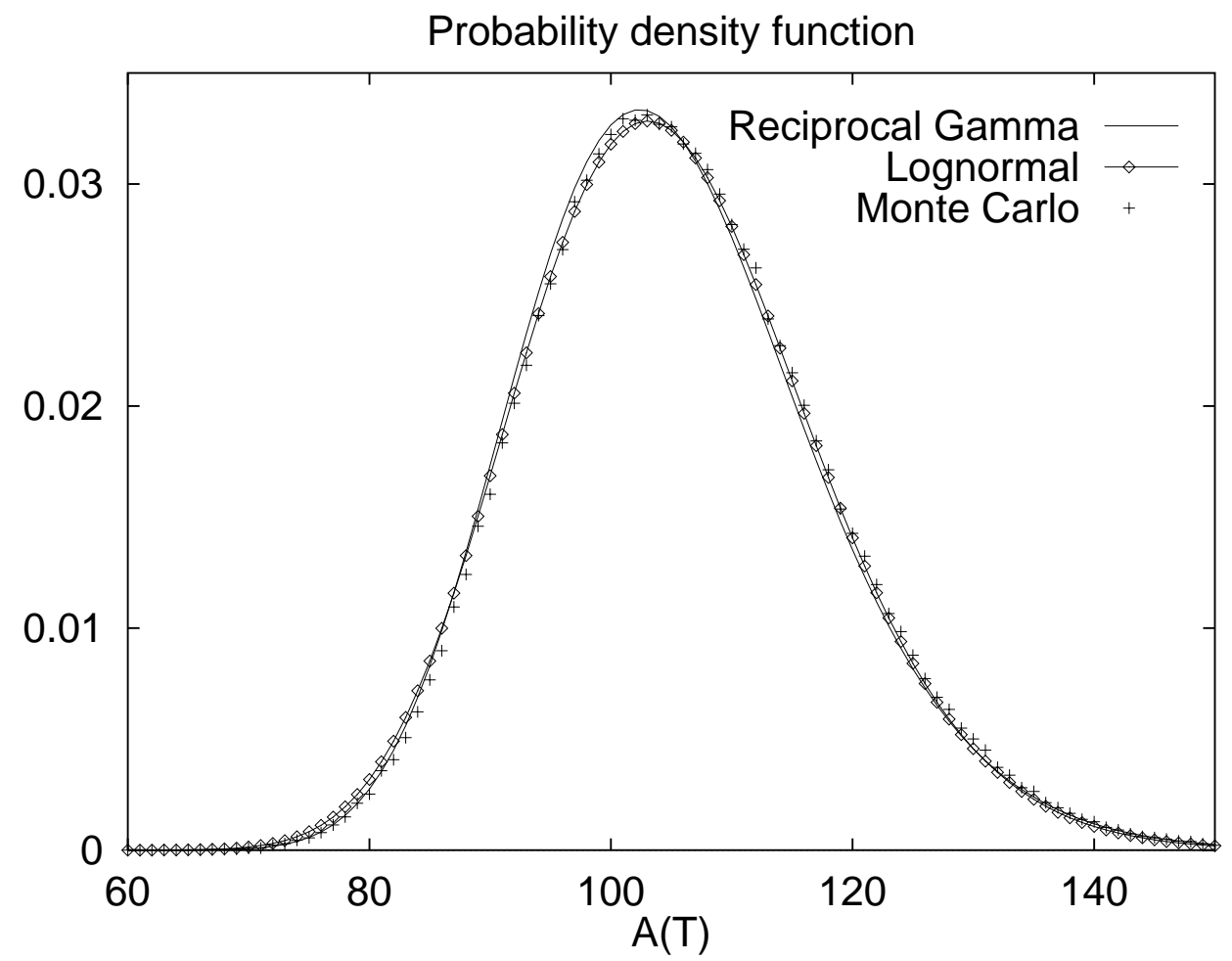

Figure 6: Risk-neutral probability density function of the arithmetic stock price average at maturity. The parameter values are: $r=0.1, q=0, \sigma=0.2, T=1, S_{0}=100$ and $n=\infty$. The expected value of the average price is 105.17 , and the variance 152.74 . For $n=\infty$ the true density function is reciprocal gamma, with parameters $\alpha=74.42$ and $\beta=1.29 \mathrm{E}-4$. This function is approximated with a lognormal distribution with the same moments. The density function is also estimated with Monte Carlo simulation, using a set of 50 runs of 10,000 paths with 1,000 time steps. 